\title{
Psychometric properties of the Polish versions of the HEXACO-60 and the HEXACO-100 personality inventories
}

\author{
Ewa Skimina (1D $1 \cdot C, D, E, E, G$, Wtodzimierz Strus (D) $1 \cdot A, B, D, E, F$, fan Cieciuch (D) $1,2 \cdot A, C, E, G$, \\ Piotr Szarota $\mathbb{D D}^{3 \cdot A, E}$, Pawet K. Izdebski $\mathbb{D D}^{4 \cdot A, E}$ \\ 1: Institute of Psychology, Cardinal Stefan Wyszyński University in Warsaw, Warsaw, Poland \\ 2: University Research Priority Program Social Networks, University of Zurich, Zurich, Switzerland \\ 3: Institute of Psychology, Polish Academy of Sciences, Warsaw, Poland \\ 4: Faculty of Psychology, Kazimierz Wielki University, Bydgoszcz, Poland
}

\section{BACKGROUND}

The HEXACO Personality Inventory-Revised (HEXACOPI-R), available in 200-, 100-, and 60-item versions, has become one of the most frequently applied measurement tools for the assessment of basic personality dimensions.

\section{PARTICIPANTS AND PROCEDURE}

In this study we examined the Polish versions of the HEXACO-60 and the HEXACO-100 inventories in a community sample of 522 individuals (aged 16-75, $M=32.02$, $S D=14.15,56.3 \%$ female). We verified the factor validity of both inventories with exploratory structural equation modeling. Additionally, we tested a six-factor solution on the HEXACO-60 items with principal axis extraction and we compared a factor matrix of the Polish adaptation of the HEXACO- 100 facets with the factor matrix of the original version of the HEXACO-100 facets in an exploratory factor comparison analysis. We analyzed correlations be- tween HEXACO domains and various models of personality traits, including the Big Two, Big Six, Big Five, and 10 Big Five aspects.

RESULTS

Internal consistency reliability coefficients for scales and subscales were satisfactory. The analyses supported the six-factor structure of the inventories and the results of correlation analyses were consistent with expectations.

CONCLUSIONS

The results indicate that the Polish versions of the HEXACO-60 and the HEXACO-100 inventories are reliable and valid instruments for measuring basic personality traits in the HEXACO model.

KEY WORDS

personality assessment; HEXACO; personality structure

CORresponding Author - Ewa Skimina, Ph.D., Institute of Psychology, Cardinal Stefan Wyszyński University

in Warsaw, 1/3 Wóycickiego Str., Building 14, 01-938 Warsaw, Poland, e-mail: e.skimina@uksw.edu.pl AUthors' CONTRIBUtion - A: Study design - B: Data collection - C: Statistical analysis - D: Data interpretation .

E: Manuscript preparation · F: Literature search · G: Funds collection

to Cite this ARTICle - Skimina, E., Strus, W., Cieciuch, J., Szarota, P., \& Izdebski, P. K. (2020). Psychometric properties of the Polish versions of the HEXACO-60 and the HEXACO-100 personality inventories. Current Issues in Personality Psychology, 8(3), 255-278.

RECEIVED 04.04.2020 • REVIEWED 23.07.2020 • ACCEPTED 30.07.2020 • PUBLISHED 19.10.2020 


\section{BACKGROUND}

For more than 20 years the predominant model of personality traits has been the Five Factor Model (FFM; McCrae \& Costa, 2003), corresponding to (and sometimes also called) the Big Five, which is derived from psycholexical research (e.g., Goldberg, 1990). The five basic traits depicted in this model are Neuroticism (vs. Emotional Stability), Extraversion, Openness to Experience (or its narrower psycholexical variant - Intellect; for a discussion on the relations between Openness to Experience and Intellect, see McCrae, 1990; Saucier, 1992; Trapnell, 1994), Agreeableness, and Conscientiousness. However, the results of psycholexical studies conducted in many languages - such as French, Dutch, Hungarian, Italian, Korean, Polish (Ashton et al., 2004), German (Ashton, Lee, Marcus, \& de Vries, 2007), Greek (Lee \& Ashton, 2009), or Croatian (Ashton \& Lee, 2008) - has challenged the five-factor structure of personality traits by revealing an additional factor, called Honesty-Humility or Honesty-Propriety (defined by terms such as sincere, honest, and modest; Ashton et al., 2004). The Big Six solution appears to be even more replicable across cultures than the Big Five, especially in languages of non-Northern European origin (e.g., Eastern European, Southern European, or Asian; Lee \& Ashton, 2008; Saucier, 2009; see Saucier, Hampson, \& Goldberg, 2000, for a discussion on the competing results of psycholexical studies).

Although the Big Six is more replicable than the Big Five, it is not fully ubiquitous among cultures. The psycholexical studies conducted so far indicate that only two factors are culturally universal (Saucier \& Srivastava, 2015; Saucier et al., 2014; Strus \& Cieciuch, 2019; Thalmayer, Saucier, Ole-Kotikash, \& Payne, 2019). The Big Two factors are usually termed Social Self-Regulation and Dynamism. Social Self-Regulation further splits into Conscientiousness, Honesty-Humility, and Agreeableness and Dynamism splits into Openness/Intellect and Extraversion. Emotionality emerges from Dynamism or from both Dynamism and Social Self-Regulation (De Raad, 2009; De Raad et al., 2010; Saucier, 2009; Saucier \& Srivastava, 2015; Saucier et al., 2014).

Polish psycholexical research has been cited as evidence of both five-factor (e.g., Peabody \& De Raad, 2002; Szarota, 1996) and six-factor (Ashton et al., 2004; Szarota, Ashton, \& Lee, 2007) solutions of basic personality dimensions. However, the latest study, conducted on a sample covering the full age range and using more extensive lexical material (Universal Dictionary of the Polish Language including 100,000 terms; the previous study was based on The Concise Polish Dictionary including 35,000 entries), supported the six-factor structure, corresponding to the content of HEXACO dimensions (Gorbaniuk, Budzińska, Owczarek, Bożek, \& Juros, 2013).
The HEXACO model (Ashton \& Lee, 2001) is currently the most popular conceptualization of the six-factor structure of personality traits. It refers to the Big Six factors as: Honesty-Humility, Emotionality, Extraversion, Agreeableness, Conscientiousness, and Openness to Experience. In turn, the most popular measure of the six factors is the HEXACO Personality Inventory-Revised (HEXACO-PI-R), available in 200-, 100-, and 60-item versions (Ashton \& Lee, 2009; Lee \& Ashton, 2004, 2018). The latter two versions have been translated into numerous languages (see http://hexaco.org/hexaco-inventory) and are widely used in personality research (Lee \& Ashton, 2018).

The HEXACO model's additional dimension, named Honesty-Humility, is not the only feature that makes it distinct from the FFM. As a consequence of extracting the sixth factor, the content of two other factors changed. The negative pole of HEXACO Agreeableness contains irritability/anger, which is covered by the positive pole of FFM Neuroticism. The positive pole of HEXACO Emotionality (a counterpart of Neuroticism) contains tenderness, which is covered by the positive pole of FFM Agreeableness. Hence, HEXACO Agreeableness and Emotionality can be understood as rotational variants of their FFM counterparts: The negative pole of HEXACO Agreeableness is rotated toward the positive pole of FFM Neuroticism and the positive pole of HEXACO Emotionality is rotated toward the positive pole of FFM Agreeableness (de Vries, Lee, \& Ashton, 2008). Extraversion, Conscientiousness, and Openness to Experience closely correspond to their FFM counterparts (Ashton \& Lee, 2007).

Lee and Ashton (2013) found that although all the FFM dimensions measured by the NEO Five-Factor Inventory (NEO-FFI) were fully explained by the set of HEXACO dimensions measured by the HEXACO-60, Honesty-Humility, Emotionality, and Agreeableness from the HEXACO model were not satisfactorily accounted for by the set of FFM dimensions. Additionally, other measures of the FFM - Big Five Aspect Scales (DeYoung, Quilty, \& Peterson, 2007), Big Five Inventory (John, Donahue, \& Kentle, 1991), Next Big Five Inventory (Soto \& John, 2017), and IPIP-50 (Goldberg, 1999) - failed to cover the variance of all HEXACO dimensions (Ashton \& Lee, 2018; Ashton, Lee, \& Visser, 2019; Ludeke et al., 2019). This indicates that the HEXACO model contains variance not shared with the FFM and gives HEXACO an advantage over FFM in terms of predicting various outcomes related to not only Honesty-Humility, but also Emotionality, and Agreeableness. Examples include relations with kin and reciprocal altruism (Ashton \& Lee, 2007), egoism (de Vries, de Vries, de Hoogh, \& Feij, 2009), prosocial behavior (Hilbig, Zettler, Leist, \& Heydasch, 2013), moral functioning (e.g., Hilbig \& Zettler, 2015), the "dark triad" traits (Lee \& Ashton, 2005), risk tak- 
ing (e.g., Ashton, Lee, Pozzebon, Visser, \& Worth, 2010), gambling (McGrath, Neilson, Lee, Rash, \& Rad, 2018), religiousness (e.g., Aghababaei, 2012; Ashton \& Lee, 2019), forgiving versus retaliating behaviors (Lee \& Ashton, 2012), guilt and shame proneness (Cohen, Wolf, Panter, \& Insko, 2011), as well as academic performance (Thalmayer, Saucier, \& Eigenhuis, 2011) and various everyday activities (Skimina, Cieciuch, \& Strus, 2018).

\section{CURRENT STUDY}

The Polish translation of the HEXACO-100 was included in a large study that confirmed the measurement invariance of this inventory across 16 languages (Thielman et al., 2019). However, the Polish version of the inventory used in that study was a pilot and not free of imperfections. The aim of this paper is twofold: (a) to introduce the refined versions of Polish translations of the HEXACO-60 and HEXACO-100, which are now available to download from the HEXACO website (http://hexaco.org/hexaco-inventory) and (b) to examine their psychometric properties, which have not yet been reported in detail.

In an initial step for this paper, we improved the Polish versions of the questionnaires modifying the items' wording on the basis of the previous results. Stylistic corrections have been made in a large number of items. They were rather subtle (e.g., changing syntax or using synonyms) and aimed at better capturing the theoretical meaning of the measured factors.

Then, we provide the detailed psychometric properties of the refined Polish versions of the HEXACO-60 and HEXACO-100 inventories as measures of six personality domains distinguished within the HEXACO model. We start with reporting descriptive statistics together with sex differences. The analyses of the original versions of the inventories revealed that women averaged substantially higher on Emotionality (large effects) and somewhat higher on Honesty-Humility (small effects; Ashton \& Lee, 2009; Lee \& Ashton, 2018). Therefore, we expected analogous results:

H1: Women score higher than men on Emotionality and Honesty-Humility.

We expected satisfactory psychometric properties of the HEXACO- 60 and the HEXACO-100 in terms of internal-consistency reliability, factor structure, and scale intercorrelations. We formulated the following hypotheses:

H2: The internal-consistency coefficients of the HEXACO-60 and HEXACO-100 scales are satisfactory and comparable to those of the original inventories and their other language adaptations.

H3: The six-factor structure of the HEXACO-60 and HEXACO-100 inventories is well fitted to the data.
H4: The pattern of HEXACO scales' intercorrelations is similar to that in studies on the original versions of the inventories and on their other language adaptations.

We examined the theoretical validity of HEXACO scales by analyzing their relationships with other measures of personality traits. We included various models: the FFM/Big Five, lexical Big Six, and Big Two. Because the HEXACO model is based on the lexical Big Six we expected that:

H5: HEXACO scales more highly correlate with their Big Six counterparts than with other Big Six scales.

Based on the similarities and differences between the HEXACO and the FFM scales, we expected that:

H6: HEXACO correlations with their FFM counterparts are higher for Extraversion, Conscientiousness, and Openness to Experience than for Emotionality and Agreeableness.

H7: FFM Agreeableness correlates with HEXACO Honesty-Humility, Emotionality, and Agreeableness at a comparable level.

Based on the results from psycholexical studies (e.g., Saucier, 2009), we formulated the following hypotheses regarding correlations between the lexical Big Two and the HEXACO scales:

H8: Big Two Dynamism correlates positively with HEXACO Extraversion and Openness to Experience, and negatively with Emotionality.

H9: Big Two Social Self-Regulation correlates positively with HEXACO Honesty-Humility, Agreeableness, and Conscientiousness.

\section{PARTICIPANTS AND PROCEDURE}

\section{PARTICIPANTS}

The study was conducted on a community sample consisting of 522 respondents, aged from 16 to 75 $(M=32.02, S D=14.15), 56.3 \%$ female ${ }^{1}$. One third of the respondents were single, $28.4 \%$ lived in a civil partnership, $29.9 \%$ were married, $2.7 \%$ were divorced, $1.7 \%$ were a widow/widower, $0.4 \%$ were separated, and $3.6 \%$ did not provide their marital status. The majority of participants indicated that their highest level of completed education was university (35.8\%) or high school (46.5\%, including current university students, constituting $18.2 \%$ of the sample). Unemployed students constituted $20.9 \%$ of the sample, other unemployed $4.0 \%$, retired persons $4.8 \%$, housewives $2.1 \%$, and the rest of the sample (68.2\%) were professionally active. The majority of participants lived in a big city (34.3\% in a city of more than 500,000 citizens; $10.5 \%$ in a city of 100,000 to 500,000 citizens), $22.6 \%$ lived in a small town (less than 100,000 citizens), and $28.9 \%$ lived in a village. 


\section{PROCEDURE}

The participants were recruited by trained research assistants (psychology students). Each of them administered the measures to approximately 6-10 respondents chosen from a pool of their distant relatives, friends, and acquaintances. Participation in the study was voluntary and anonymous.

The study complied with the recommendations of the Commission of Ethics and Bioethics at the Cardinal Stefan Wyszyński University in Warsaw (participants were informed that participation in the study was voluntary and they provided their oral consent). Because the study was conducted on adults and included only self-report measures of personality traits it did not require formal approval by the Commission of Ethics and Bioethics, according to the institutional guidelines.

The study was carried out using a self-report paper-and-pencil method in three sessions at approximately 2-4-week intervals as part of a larger, four-session research project. The Big Five Inventory and the Big Five Aspect Scales were filled out during the first session, the Questionnaire Big Six during the third session, and the HEXACO-100 during the fourth session. The rest of the measures administered during the sessions (including the second one) were other personality questionnaires (not relevant to this study), and for this reason it was expected that they would not significantly impact the results of the current study.

\section{MEASURES}

HEXACO-100. The HEXACO-100 is a 100 -item questionnaire operationalization of the HEXACO model of personality traits (Lee \& Ashton, 2018). Ninetysix items are distributed throughout six scales measuring six basic HEXACO dimensions (16 items per scale), with each scale composed of four facet scales (four items per facet scale; see Lee \& Ashton, 2004, for definitions). Four additional items constitute an interstitial facet of Altruism (approximately equally correlated with Honesty-Humility, Agreeableness, and Emotionality). In this study we used a refined version of Polish translations of the HEXACO-100 items (see Current study, para. 2), which we present in the Appendix. Participants indicate their answers on a 5-point Likert scale from 1 (strongly disagree) to 5 (strongly agree). Alpha reliabilities of original English versions of HEXACO-100 scales ranged from .81 for Openness to Experience to .85 for Extraversion, with a mean of .83 , at the basic dimension level and from .52 for Unconventionality to .81 for Greed Avoidance, with a mean of .70, at the facet level.

HEXACO-60. The HEXACO-60 is a shortened version of the HEXACO Personality Inventory (Ashton
\& Lee, 2009). It consists of 60 items selected from the HEXACO-100 and assesses the six HEXACO dimensions (10 items per scale). The HEXACO-60 is not recommended for assessing personality facets. The response scale is the same as in the HEXACO- 100 . Alpha reliabilities of original English versions of HEXACO-60 scales in a community sample ranged from .73 for Emotionality and Extraversion to .80 for Openness to Experience, with a mean of .75. To assess psychometric properties of the HEXACO-60 we selected relevant items from the HEXACO-100 (the same that constitute the original version of the HEXACO-60; Ashton \& Lee, 2009).

Big Five Aspect Scales. The Big Five Aspect Scales (BFAS) is a measure of Big Five personality traits (DeYoung et al., 2007). It consists of 100 items, derived from the International Personality Item Pool (IPIP; Goldberg, 1999; Goldberg et al., 2006), assessing the five dimensions (20 items per scale) and their 10 aspects (10 items per subscale). The scales (and subscales) are: Neuroticism (Volatility and Withdrawal), Agreeableness (Compassion and Politeness), Conscientiousness (Industriousness and Orderliness), Extraversion (Enthusiasm and Assertiveness), and Openness/Intellect (Intellect and Openness to Experience). Participants indicate their answers on a 5-point Likert scale from 1 (very inaccurately describes me) to 5 (very accurately describes me). We used a Polish translation of the BFAS, prepared by Strus, Cieciuch, and Rowinski (2012). In the current study, Cronbach's $\alpha$ coefficients for domains ranged from .84 for Conscientiousness to .91 for Neuroticism, with a mean of .87 ; for the aspect scales they ranged from .75 for Compassion to .88 for Volatility, with a mean of .82 .

Big Five Inventory. The Big Five Inventory (BFI) was developed as a short measure of the Big Five dimensions (John, Naumann, \& Soto, 2008). It contains 44 brief items (eight to 10 items per scale), based on the trait adjectives known to be prototypical markers of the Big Five. Participants indicate their answers on a 5-point Likert scale from 1 (disagree strongly) to 5 (agree strongly). We used a Polish version of the BFI (Strus \& Cieciuch, 2019), and in the current study, Cronbach's $\alpha$ coefficients for dimensions ranged from .74 for Agreeableness to .82 for Neuroticism, with a mean of .79 .

Questionnaire Big Six. The Questionnaire Big Six (QB6), consisting of 40 items, is a measure of three models of personality traits derived from psycholexical studies: the Big Six, Big Five, and Big Two (Thalmayer \& Saucier, 2014). Participants indicate their answers on a 5-point Likert scale from 1 (very inaccurately describes me) to 5 (very accurately describes me). We used a Polish version of the QB6 (Strus $\&$ Cieciuch, 2019) to measure two models: the Big Six and Big Two (we did not use it for measuring the Big Five scales because they were measured in this study by two well-validated instruments described above). 
The Big Six measure is composed of the following scales: Conscientiousness, Agreeableness, Honesty/ Propriety, Extraversion, Originality (equivalent of Openness to Experiences), and Resiliency (equivalent of Emotionality). Each scale constitutes five items, which gives 30 items in total. In the current study, Cronbach's $\alpha$ coefficients for the Big Six scales ranged from .68 for Conscientiousness to .78 for Resiliency, with a mean of .71.

The Big Two model corresponds to the first two factors found in nine diverse psycholexical studies (Saucier et al., 2014). It should not be mistaken for higher-order factors derived from the Big Five scales. The two basic dimensions are Social Self-Regulation (internalization of social and cultural norms) and Dynamism (the relative proportion of approach vs. avoidant tendencies in personality; Thalmayer \& Saucier, 2014). Each scale contains seven items, which gives 14 items in total. In the current study, Cronbach's $\alpha$ coefficients were .68 for Social SelfRegulation and .69 for Dynamism.

\section{ANALYSES}

Descriptive statistics and reliability ( $\mathrm{H} 1$ and $\mathrm{H} 2)$. We assessed means and standard deviations for the HEXACO-60 scales, as well as the HEXACO-100 scales and subscales, separately for men and for women. Gender differences were assessed by Student's $t$ test. The internal consistency was assessed by calculating mean inter-item correlations, mean itemtotal correlations, and Cronbach's $\alpha$ coefficients for all scales and subscales. In this respect, our analyses were analogous to those conducted on the original versions of the HEXACO-60 and HEXACO-100 inventories (Ashton \& Lee, 2009; Lee \& Ashton, 2018).

Additionally, we calculated $\omega$ coefficients introduced by McDonald $(1978,1999)$ and based on a factor analysis. They lead to a more accurate correction for attenuation than Cronbach's $\alpha$, especially in the case of multidimensional scales (Revelle \& Zinbarg, 2009). McDonald distinguished two coefficients: omega total $\left(\omega_{\mathrm{t}}\right.$, based on the sum of squared loadings on all factors; McDonald, 1978) and omega hierarchical $\left(\omega_{h}\right.$, based on the sum of the squared loadings on the general factor; McDonald, 1999). Omega hierarchical has been renamed omega general $\left(\omega_{g}\right)$ to reflect that the statistic is an estimate of the percentage of variance of a scale due to a general factor (Condon \& Revelle, 2014). When several dimensions contribute to the prediction of the criterion of interest, $\omega_{t}$ will lead to a more accurate correction for attenuation and when only one, general factor contributes to the prediction of the criterion of interest, $\omega_{\mathrm{g}}$ will be more accurate (Revelle \& Zinbarg, 2009). If a scale is unidimensional, there is no difference between $\omega_{t}$ and $\omega_{g}$ coefficients. We calculated both coefficients $\left(\omega_{t}\right.$ and $\omega_{g}$ ) for six domain scales measured by the HEXACO-60 and the HEXACO-100 because each of them is composed of four dimensions. This way we estimated what part of each basic scale variance is due to its general factor (e.g., a general factor of Honesty-Humility) - this has not been investigated in previous studies.

We used the omega function in the psych package in $\mathrm{R}$ for calculating $\alpha$ and $\omega$ coefficients (Revelle, 2016), SPSS Statistics 24 for calculating means, standard deviations, Student's $t$, mean interitem correlations, and mean item-total correlations as well as Becker's (1998) Effect Size Calculators for calculating Cohen's $d$ for gender differences.

Factor analysis (H3). To examine the factorial structure of the Polish adaptation of the HEXACO-60, we first followed the procedure applied by the authors (Ashton \& Lee, 2009). Namely, using SPSS Statistics 24, we conducted principal axis extraction with varimax rotation of six factors. Additionally, we examined a six-factor solution in exploratory structural equation modeling (ESEM) with target rotation, using Mplus software. This procedure has not been applied in previous research on the HEXACO inventories despite its advantages.

ESEM combines the strengths of exploratory factor analysis (EFA) and confirmatory factor analysis (CFA), which makes it suitable to test the internal structure of a personality inventory. EFA is considered an "exploratory," data-driven approach, as it does not require any explicit a priori assumptions regarding the number of factors and it allows for cross-loadings. CFA is considered "confirmatory," as it requires a priori specification of the number of factors and indicators meaningfully loading on the stipulated factors. CFA is very restrictive, fixing all cross-loadings at zero. As a result, models tested in CFA are unlikely to be suitable for data collected with personality inventories, which often have many small cross-loadings that are motivated both by the theory and the formulation of the measurement (Asparouhov \& Muthén, 2009). Hence, personality inventories perform poorly in CFA and using this procedure to verify their internal structure is not recommended (Hopwood \& Donnellan, 2010). ESEM is a useful alternative because, like CFA, it can be used as a confirmatory analysis, with the number of factors and expected loadings of indicators on these factors specified a priori, and it gives access to all the usual SEM parameters. At the same time, as with EFA, it allows for cross-loadings. Because it is less restrictive than CFA, it is more suitable for models of personality traits.

In target rotation, cross-loadings are specified as zero but they are not fixed as in CFA. The loadings change during rotation to find a good fit. They are made as close to zero as possible, but they may be large if it is necessary to provide good fit (Browne, 2001). 
We used the same procedure - ESEM with target rotation - to test the six-factor solution of the HEXACO-100 on the facet level. In the last step, we examined the congruence between factorial structures of the Polish and original versions of the HEXACO-100 in an exploratory factor comparison analysis using the orthogonal non-Procrustes (i.e., unadjusted, without row normalization) procedure by Barrett (2013). This procedure allows one to compare the target and comparison matrices using congruence coefficients which quantify the similarity of the target and comparison loading matrices for overall solution congruence, for each factor (column) and for each row (variable) in the matrices. As a target we used the loadings matrix of HEXACO-100 facet scales on six varimax-rotated components (results from a principal component analysis - PCA), published by Lee and Ashton (2018, p. 548) and obtained on the basis of selfreports from an online sample $(N=100,318)$. The comparison matrix was obtained for our data $(N=522)$ in the same way as the target matrix, that is, in a PCA with varimax rotation conducted on 25 facets. Then the results were rotated using the non-Procrustes procedure, where the comparison matrix is aligned to the target matrix by orthogonal rotation of the first one against the second to minimize the sum of squared deviations between the two matrices. Components are not aligned one by one, but a fixed orthogonal structure is maintained across all dimensions. Using unadjusted rotation (without row normalization) does not affect the relative positions of the variables in the common space. The analysis was conducted in the Orthosim 2.1 software by Barrett (2013).

Correlation analyses (H4-H9). We used Pearson's $r$ test to examine (a) correlations among the HEXACO-60 and the HEXACO-100 scales, (b) correlations of the six HEXACO domains measured by both HEXACO inventories with measures of the Big Five, Big Six, and Big Two, and (c) correlations of the HEXACO domains and facets with the Big Five aspects.

\section{RESULTS}

\section{DESCRIPTIVE STATISTICS AND RELIABILITY (H1 AND H2)}

In Table 1, descriptive statistics are presented with $\alpha$ and $\omega$ reliability coefficients, and gender differences in the HEXACO-60 and the HEXACO-100 scales. For many HEXACO scales gender differences were statistically significant. According to Cohen's $d$ test, large effects were found only for Emotionality and its facets (Fearfulness, Anxiety, and Sentimentality) - women scored higher on these scales than men. There was a medium difference between women and men in Altruism (women's mean was higher). Women also scored higher than men on Honesty-Humility; this effect was small, however. These findings are consistent with previous research and confirm $\mathrm{H} 1$ (Ashton \& Lee, 2009; Lee \& Ashton, 2018).

Table 1

Descriptive statistics and reliability coefficients for the HEXACO-60 and the HEXACO-100 scales

\begin{tabular}{|c|c|c|c|c|c|c|c|c|}
\hline \multirow[t]{2}{*}{ HEXACO scale } & \multirow{2}{*}{$\begin{array}{l}\text { Mean } \\
\text { inter- } \\
\text { item } r\end{array}$} & \multirow{2}{*}{$\begin{array}{l}\text { Mean } \\
\text { item- } \\
\text { total } r\end{array}$} & \multirow[t]{2}{*}{$\alpha$} & \multirow[t]{2}{*}{$\omega_{t}\left(\omega_{g}\right)$} & \multirow{2}{*}{$\begin{array}{l}\text { Women } \\
n=294 \\
M(\mathrm{SD})\end{array}$} & \multirow{2}{*}{$\begin{array}{c}\text { Men } \\
n=228 \\
M(\mathrm{SD})\end{array}$} & \multicolumn{2}{|c|}{$\begin{array}{c}\text { Difference between } \\
\text { women and men }\end{array}$} \\
\hline & & & & & & & $\begin{array}{c}\text { Student's } \\
t\end{array}$ & $\begin{array}{c}\text { Cohen's } \\
d\end{array}$ \\
\hline \multicolumn{9}{|l|}{ HEXACO-60 basic scales } \\
\hline Honesty-Humility-60 & .26 & .45 & .78 & $\begin{array}{c}.84 \\
(.58)\end{array}$ & $\begin{array}{c}3.61 \\
(0.60)\end{array}$ & $\begin{array}{c}3.38 \\
(0.67)\end{array}$ & $4.03^{* *}$ & 0.36 \\
\hline Emotionality-60 & .28 & .47 & .80 & $\begin{array}{l}.86 \\
(.64)\end{array}$ & $\begin{array}{c}3.50 \\
(0.56)\end{array}$ & $\begin{array}{c}2.76 \\
(0.55)\end{array}$ & $15.16^{* * *}$ & 1.33 \\
\hline Extraversion-60 & .31 & .50 & .82 & $\begin{array}{c}.87 \\
(.65)\end{array}$ & $\begin{array}{c}3.36 \\
(0.61)\end{array}$ & $\begin{array}{c}3.44 \\
(0.63)\end{array}$ & -1.46 & -0.13 \\
\hline Agreeableness- 60 & .25 & .43 & .77 & $\begin{array}{c}.84 \\
(.58)\end{array}$ & $\begin{array}{c}3.09 \\
(0.63)\end{array}$ & $\begin{array}{c}3.03 \\
(0.55)\end{array}$ & 1.08 & 0.10 \\
\hline Conscientiousness-60 & .29 & .48 & .80 & $\begin{array}{c}.85 \\
(.64)\end{array}$ & $\begin{array}{c}3.65 \\
(0.58)\end{array}$ & $\begin{array}{c}3.54 \\
(0.59)\end{array}$ & $2.17^{*}$ & 0.19 \\
\hline $\begin{array}{l}\text { Openness to } \\
\text { Experience- } 60\end{array}$ & .26 & .45 & .78 & $\begin{array}{l}.83 \\
(.67)\end{array}$ & $\begin{array}{c}3.33 \\
(0.66)\end{array}$ & $\begin{array}{c}3.33 \\
(0.66)\end{array}$ & -0.01 & 0.00 \\
\hline
\end{tabular}

(Table 1 continues) 
Table 1

(Table 1 continued)

\begin{tabular}{|c|c|c|c|c|c|c|c|c|}
\hline \multirow[t]{2}{*}{ HEXACO scale } & \multirow{2}{*}{$\begin{array}{l}\text { Mean } \\
\text { inter- } \\
\text { item } r\end{array}$} & \multirow{2}{*}{$\begin{array}{l}\text { Mean } \\
\text { item- } \\
\text { total } r\end{array}$} & \multirow[t]{2}{*}{$\alpha$} & \multirow[t]{2}{*}{$\omega_{t}\left(\omega_{g}\right)$} & \multirow{2}{*}{$\begin{array}{c}\text { Women } \\
n=294 \\
M(\mathrm{SD})\end{array}$} & \multirow{2}{*}{$\begin{array}{c}\text { Men } \\
n=228 \\
M(\mathrm{SD})\end{array}$} & \multicolumn{2}{|c|}{$\begin{array}{c}\text { Difference between } \\
\text { women and men }\end{array}$} \\
\hline & & & & & & & $\begin{array}{c}\text { Student's } \\
t\end{array}$ & $\begin{array}{c}\text { Cohen's } \\
d\end{array}$ \\
\hline \multicolumn{9}{|l|}{ HEXACO-100 basic scales } \\
\hline Honesty-Humility-100 & .26 & .47 & .85 & $\begin{array}{c}.88 \\
(.65)\end{array}$ & $\begin{array}{c}3.60 \\
(0.57)\end{array}$ & $\begin{array}{c}3.42 \\
(0.64)\end{array}$ & $3.35^{* *}$ & 0.30 \\
\hline Emotionality-100 & .26 & .47 & .85 & $\begin{array}{c}.88 \\
(.61)\end{array}$ & $\begin{array}{c}3.50 \\
(0.52)\end{array}$ & $\begin{array}{c}2.81 \\
(0.51)\end{array}$ & $15.03^{* * *}$ & 1.34 \\
\hline Extraversion-100 & .28 & .50 & .86 & $\begin{array}{l}.89 \\
(.66)\end{array}$ & $\begin{array}{c}3.42 \\
(0.57)\end{array}$ & $\begin{array}{c}3.48 \\
(0.58)\end{array}$ & -1.13 & -0.10 \\
\hline Agreeableness-100 & .24 & .45 & .84 & $\begin{array}{l}.87 \\
(.59)\end{array}$ & $\begin{array}{c}2.93 \\
(0.58)\end{array}$ & $\begin{array}{c}2.89 \\
(0.52)\end{array}$ & 0.73 & 0.07 \\
\hline Conscientiousness-100 & .27 & .48 & .85 & $\begin{array}{l}.88 \\
(.64)\end{array}$ & $\begin{array}{c}3.66 \\
(0.55)\end{array}$ & $\begin{array}{c}3.58 \\
(0.56)\end{array}$ & 1.67 & 0.14 \\
\hline $\begin{array}{l}\text { Openness to } \\
\text { Experience-100 }\end{array}$ & .25 & .46 & .84 & $\begin{array}{c}.88 \\
(.57)\end{array}$ & $\begin{array}{c}3.32 \\
(0.63)\end{array}$ & $\begin{array}{c}3.37 \\
(0.62)\end{array}$ & -0.80 & -0.08 \\
\hline \multicolumn{9}{|l|}{ HEXACO-100 facets } \\
\hline H_Sincerity & .35 & .46 & .68 & .69 & $\begin{array}{c}3.49 \\
(0.77)\end{array}$ & $\begin{array}{l}3.46 \\
(0.79)\end{array}$ & 0.47 & 0.04 \\
\hline H_Fairness & .42 & .55 & .75 & .76 & $\begin{array}{c}3.85 \\
(0.83)\end{array}$ & $\begin{array}{l}3.52 \\
(0.96)\end{array}$ & $4.21^{* * *}$ & 0.37 \\
\hline H_Greed Avoidance & .47 & .59 & .78 & .79 & $\begin{array}{c}3.33 \\
(0.87)\end{array}$ & $\begin{array}{c}3.16 \\
(0.92)\end{array}$ & $2.12^{*}$ & 0.19 \\
\hline H_Modesty & .30 & .42 & .64 & .64 & $\begin{array}{c}3.73 \\
(0.65)\end{array}$ & $\begin{array}{l}3.55 \\
(0.71)\end{array}$ & $3.03^{* *}$ & 0.26 \\
\hline E_Fearfulness & .39 & .51 & .72 & .73 & $\begin{array}{c}3.20 \\
(0.74)\end{array}$ & $\begin{array}{c}2.38 \\
(0.74)\end{array}$ & $12.41^{* * *}$ & 1.11 \\
\hline E_Anxiety & .25 & .35 & .57 & .58 & $\begin{array}{c}3.59 \\
(0.68)\end{array}$ & $\begin{array}{l}3.03 \\
(0.68)\end{array}$ & $9.33^{* * *}$ & 0.82 \\
\hline E_Dependence & .46 & .58 & .77 & .78 & $\begin{array}{c}3.44 \\
(0.83)\end{array}$ & $\begin{array}{c}2.81 \\
(0.76)\end{array}$ & $8.97^{* * *}$ & 0.79 \\
\hline E_Sentimentality & .39 & .50 & .71 & .72 & $\begin{array}{c}3.75 \\
(0.70)\end{array}$ & $\begin{array}{c}3.04 \\
(0.68)\end{array}$ & $11.81^{* * *}$ & 1.03 \\
\hline X_Social Self-Esteem & .34 & .46 & .68 & .68 & $\begin{array}{c}3.53 \\
(0.69)\end{array}$ & $\begin{array}{l}3.64 \\
(0.66)\end{array}$ & -1.85 & -0.16 \\
\hline X_Social Boldness & .41 & .52 & .73 & .73 & $\begin{array}{c}3.03 \\
(0.79)\end{array}$ & $\begin{array}{c}3.22 \\
(0.81)\end{array}$ & $-2.65^{* *}$ & -0.22 \\
\hline X_Sociability & .28 & .40 & .60 & .65 & $\begin{array}{c}3.63 \\
(0.65)\end{array}$ & $\begin{array}{c}3.47 \\
(0.71)\end{array}$ & $2.50^{*}$ & 0.24 \\
\hline X_Liveliness & .48 & .59 & .79 & .79 & $\begin{array}{c}3.49 \\
(0.82)\end{array}$ & $\begin{array}{c}3.58 \\
(0.72)\end{array}$ & -1.23 & -0.11 \\
\hline A_Forgiveness & .44 & .57 & .76 & .77 & $\begin{array}{c}2.72 \\
(0.83)\end{array}$ & $\begin{array}{c}2.61 \\
(0.76)\end{array}$ & 1.66 & 0.14 \\
\hline
\end{tabular}

(Table 1 continues) 
Table 1

(Table 1 continued)

\begin{tabular}{|c|c|c|c|c|c|c|c|c|}
\hline \multirow[t]{2}{*}{ HEXACO scale } & \multirow{2}{*}{$\begin{array}{l}\text { Mean } \\
\text { inter- } \\
\text { item r }\end{array}$} & \multirow{2}{*}{$\begin{array}{l}\text { Mean } \\
\text { item- } \\
\text { total r }\end{array}$} & \multirow[t]{2}{*}{$\alpha$} & \multirow[t]{2}{*}{$\omega_{t}\left(\omega_{g}\right)$} & \multirow{2}{*}{$\begin{array}{l}\text { Women } \\
n=294 \\
M(\mathrm{SD})\end{array}$} & \multirow{2}{*}{$\begin{array}{c}\text { Men } \\
n=228 \\
M(\mathrm{SD})\end{array}$} & \multicolumn{2}{|c|}{$\begin{array}{c}\text { Difference between } \\
\text { women and men }\end{array}$} \\
\hline & & & & & & & $\begin{array}{c}\text { Student's } \\
t\end{array}$ & $\begin{array}{c}\text { Cohen's } \\
d\end{array}$ \\
\hline A_Gentleness & .34 & .45 & .67 & .67 & $\begin{array}{l}3.37 \\
(0.71)\end{array}$ & $\begin{array}{l}3.30 \\
(0.67)\end{array}$ & 1.28 & 0.10 \\
\hline A_Flexibility & .28 & .38 & .60 & .61 & $\begin{array}{l}2.82 \\
(0.75)\end{array}$ & $\begin{array}{l}2.70 \\
(0.64)\end{array}$ & 1.91 & 0.17 \\
\hline A_Patience & .36 & .47 & .69 & .69 & $\begin{array}{l}2.79 \\
(0.74)\end{array}$ & $\begin{array}{c}2.96 \\
(0.77)\end{array}$ & $-2.58^{*}$ & -0.23 \\
\hline C_Organization & .39 & .51 & .72 & .73 & $\begin{array}{l}3.84 \\
(0.79)\end{array}$ & $\begin{array}{l}3.63 \\
(0.79)\end{array}$ & $3.09^{* *}$ & 0.27 \\
\hline C_Diligence & .38 & .50 & .71 & .71 & $\begin{array}{l}3.78 \\
(0.66)\end{array}$ & $\begin{array}{l}3.83 \\
(0.68)\end{array}$ & -0.91 & -0.07 \\
\hline C_Perfectionism & .33 & .44 & .67 & .67 & $\begin{array}{l}3.64 \\
(0.70)\end{array}$ & $\begin{array}{l}3.49 \\
(0.67)\end{array}$ & $2.51^{*}$ & 0.22 \\
\hline C_Prudence & .36 & .48 & .69 & .70 & $\begin{array}{l}3.38 \\
(0.72)\end{array}$ & $\begin{array}{l}3.37 \\
(0.70)\end{array}$ & 0.21 & 0.01 \\
\hline $\begin{array}{l}\text { O_Aesthetic } \\
\text { Appreciation }\end{array}$ & .37 & .49 & .70 & .70 & $\begin{array}{l}3.46 \\
(0.87)\end{array}$ & $\begin{array}{l}3.12 \\
(0.89)\end{array}$ & $4.42^{* * *}$ & 0.39 \\
\hline O_Inquisitiveness & .38 & .50 & .71 & .71 & $\begin{array}{l}3.12 \\
(0.87)\end{array}$ & $\begin{array}{l}3.50 \\
(0.85)\end{array}$ & $-4.95^{* * *}$ & -0.44 \\
\hline O_Creativity & .39 & .51 & .72 & .73 & $\begin{array}{l}3.45 \\
(0.83)\end{array}$ & $\begin{array}{l}3.46 \\
(0.79)\end{array}$ & -0.14 & -0.01 \\
\hline O_Unconventionality & .23 & .33 & .55 & .56 & $\begin{array}{l}3.27 \\
(0.64)\end{array}$ & $\begin{array}{c}3.39 \\
(0.65)\end{array}$ & $-2.27^{*}$ & -0.18 \\
\hline Altruism & .26 & .37 & .59 & .59 & $\begin{array}{l}3.91 \\
(0.59)\end{array}$ & $\begin{array}{c}3.50 \\
(0.62)\end{array}$ & $7.79 * * *$ & 0.68 \\
\hline
\end{tabular}

Note. $N=522 ; \omega_{\mathrm{t}}$ - omega total (the total amount of reliable variance in the scale); $\omega_{\mathrm{g}}$ - omega general (the percentage of the scale variance due to the general factor) $;{ }^{*} p<.05,{ }^{* *} p<.01,{ }^{* * *} p<.001$.

At the facet level one can note some more significant gender differences. For instance, men are higher on Social Boldness and women are higher on Sociability, whereas at the domain level men and women do not differ in Extraversion. Similarly, they do not differ in Openness to Experience; however, women are higher on Aesthetic Appreciation and men are higher on Inquisitiveness and Unconventionality.

The reliability coefficients are satisfactory, confirming H2. Specifically, Cronbach's $\alpha$ for the HEXACO-60 basic scales ranges from .77 (Agreeableness) to .82 (Extraversion) with a mean of .79. Cronbach's $\alpha$ for the HEXACO-100 basic scales ranges from 84 (Agreeableness and Openness to Experience) to .86 (Extraversion), with a mean of .85 , and for the HEXACO- 100 facets $\alpha$ ranges from .55 (Unconventionality) to .79 (Liveliness), with a mean of .69. The results are similar to those re- ported for the original versions of both inventories (see Ashton \& Lee, 2009, Table 2; Lee \& Ashton, 2018, Table 1). Omegas for unidimensional HEXACO-100 facet scales are only slightly higher than alphas and range from .56 to .79 with a mean of .70. For the HEXACO-60 and the HEXACO-100 six-factor scales we computed both $\omega_{\mathrm{t}}$ and $\omega_{\mathrm{g}}$ coefficients. Because they are multidimensional, $\omega_{t}$ coefficients should be the most accurate for assessing their reliability. For the HEXACO-60 they ranged from .83 (Openness to Experience) to .87 (Extraversion) with a mean of .85, and for the HEXACO-100 from .87 (Agreeableness) to .89 (Extraversion) with a mean of .88 , showing high reliability. Omega general coefficients are lower, indicating that distinguishing four subscales is justifiable. However, general factor saturation might be considered relatively high, especially in comparison with results obtained by Wilt 
and Revelle (2019) for the Big Five scales. In this study, $\omega_{\mathrm{g}} \mathrm{s}$ for the six HEXACO-60 scales ranged from .58 (Honesty-Humility and Agreeableness) to .67 (Openness to Experience) with a mean of .63, and for the six HEXACO-100 scales from .57 (Openness to Experience) to .66 (Extraversion) with a mean of .62; in Wilt and Revelle's study they ranged from .33 (Conscientiousness) to .59 (Extraversion) with a mean of .49 .

\section{FACTOR STRUCTURE (H3)}

HEXACO-60. The six-factor model was tested on the HEXACO-60 items in two analyses: principal axis factoring (PAF) with varimax rotation and ESEM with target rotation (cross-loadings specified to be close to zero). In PAF, the first six common factors accounted for $39.7 \%$ of the item variance (compared to $37.4 \%$ and $29.1 \%$ reported for the original English version; Ashton \& Lee, 2009). The eigenvalues of the first 10 factors were $6.19,5.88,3.55,3.24,2.92,2.04,1.77,1.58,1.43$, and 1.33. All primary loadings and only six cross-loadings exceeded .30. One item (Item 61: "People have often told me that I have a good imagination") from the Openness to Experience scale had a higher cross-loading on Extraversion than its primary loading. One item (Item 32: "I often push myself very hard when trying to achieve a goal") from the Conscientiousness scale had a cross-loading on Extraversion equal to its primary loading. All factor loadings are presented in Table 2.

Table 2

Factor loadings from PAF and ESEM conducted on the HEXACO-60 items

\begin{tabular}{|c|c|c|c|c|c|c|c|c|c|c|c|c|}
\hline \multirow[t]{2}{*}{ Item } & \multicolumn{6}{|c|}{ PAF } & \multicolumn{6}{|c|}{ ESEM } \\
\hline & $\mathrm{H}$ & $\mathrm{E}$ & $X$ & A & $\mathrm{C}$ & $\mathrm{O}$ & $\mathrm{H}$ & $\mathrm{E}$ & $x$ & A & $\mathrm{C}$ & $\mathrm{O}$ \\
\hline 30 & -.40 & -.01 & -.10 & .04 & -.12 & .00 & -.36 & -.03 & -.11 & -.02 & -.10 & .04 \\
\hline 54 & .52 & .03 & .04 & -.04 & .08 & .08 & .49 & .05 & .05 & .07 & -.05 & .04 \\
\hline 78 & -.52 & -.09 & -.03 & .03 & -.13 & -.04 & -.45 & -.11 & -.06 & -.04 & -.11 & -.01 \\
\hline 12 & .43 & -.11 & -.08 & -.31 & .13 & .11 & .49 & -.09 & -.10 & -.16 & .04 & .07 \\
\hline 60 & -.36 & .09 & .03 & .14 & -.16 & -.06 & -.35 & .08 & .03 & .04 & -.12 & -.03 \\
\hline 84 & .48 & -.10 & -.08 & -.24 & .16 & .11 & .53 & -.08 & -.09 & -.07 & .07 & .06 \\
\hline 18 & -.36 & .10 & -.07 & .31 & -.02 & .00 & -.39 & .07 & -.03 & .19 & .04 & .02 \\
\hline 90 & .50 & .07 & .18 & -.28 & .03 & .07 & .57 & .12 & .17 & -.12 & -.06 & .04 \\
\hline 72 & .47 & -.08 & .00 & -.11 & .06 & -.05 & .49 & -.06 & -.02 & .01 & .02 & -.09 \\
\hline 96 & .60 & -.06 & .12 & -.21 & .01 & -.07 & .68 & -.01 & .08 & -.02 & -.05 & -.12 \\
\hline 5 & .10 & .47 & -.08 & .15 & -.06 & .12 & .09 & .48 & -.02 & .11 & -.07 & .10 \\
\hline 53 & .06 & .57 & -.21 & .15 & .00 & .11 & .09 & .57 & -.15 & .12 & -.03 & .07 \\
\hline 77 & -.02 & -.54 & .21 & .08 & -.04 & -.20 & -.01 & -.54 & .15 & .14 & .02 & -.19 \\
\hline 11 & .00 & .51 & -.24 & -.04 & -.09 & .00 & .04 & .51 & -.23 & -.08 & -.13 & -.02 \\
\hline 35 & .18 & -.31 & .12 & -.01 & .08 & .16 & .15 & -.30 & .13 & .06 & .08 & .16 \\
\hline 17 & .02 & .55 & .14 & .06 & .01 & .07 & .07 & .57 & .19 & .05 & -.01 & .04 \\
\hline 41 & .05 & -.57 & .14 & .00 & -.10 & -.05 & .02 & -.57 & .09 & .04 & -.07 & -.02 \\
\hline 23 & -.08 & .63 & .09 & .19 & .01 & -.05 & -.08 & .63 & .14 & .12 & .03 & -.07 \\
\hline 71 & -.07 & .55 & .06 & -.01 & -.10 & -.04 & -.03 & .57 & .09 & -.06 & -.12 & -.04 \\
\hline 95 & .15 & -.54 & -.05 & -.03 & .01 & .16 & .15 & -.55 & -.08 & .06 & .01 & .17 \\
\hline 4 & .10 & -.15 & .52 & .05 & -.20 & .08 & .12 & -.10 & .51 & .10 & -.19 & .10 \\
\hline 52 & -.07 & .15 & -.55 & .07 & .02 & .06 & -.03 & .11 & -.53 & .07 & .00 & .05 \\
\hline 76 & -.02 & .29 & -.50 & -.11 & .29 & .01 & -.06 & .25 & -.47 & -.15 & .26 & -.01 \\
\hline 10 & -.02 & .10 & -.51 & .27 & .04 & .16 & .04 & .07 & -.46 & .30 & .04 & .12 \\
\hline
\end{tabular}


Table 2

(Table 2 continued)

\begin{tabular}{|c|c|c|c|c|c|c|c|c|c|c|c|c|}
\hline \multirow[t]{2}{*}{ Item } & \multicolumn{6}{|c|}{ PAF } & \multicolumn{6}{|c|}{ ESEM } \\
\hline & $\mathrm{H}$ & $\mathrm{E}$ & $X$ & A & $\mathrm{C}$ & $\mathrm{O}$ & $\mathrm{H}$ & $\mathrm{E}$ & $X$ & A & $\mathrm{C}$ & $\mathrm{O}$ \\
\hline 34 & .08 & -.04 & .63 & -.04 & -.01 & -.18 & .04 & -.01 & .60 & -.04 & .03 & -.17 \\
\hline 58 & .21 & -.01 & .42 & -.12 & .08 & -.09 & .15 & .02 & .41 & -.10 & .09 & -.10 \\
\hline 64 & -.02 & .14 & .55 & .11 & .00 & .00 & -.01 & .18 & .57 & .10 & .04 & .00 \\
\hline 88 & .10 & .12 & .57 & .15 & .02 & .00 & .09 & .16 & .60 & .16 & .06 & .00 \\
\hline 46 & -.06 & -.04 & .64 & .15 & -.13 & .07 & -.04 & .00 & .66 & .15 & -.09 & .10 \\
\hline 94 & .01 & .17 & -.61 & .12 & .12 & .05 & .06 & .13 & -.59 & .14 & .09 & .01 \\
\hline 3 & -.13 & .02 & .09 & .54 & .05 & -.02 & -.15 & -.01 & .14 & .49 & .14 & -.02 \\
\hline 27 & -.16 & .07 & .10 & .59 & .01 & -.03 & -.16 & .05 & .15 & .53 & .11 & -.04 \\
\hline 9 & .22 & -.02 & -.07 & -.44 & .05 & -.06 & .13 & -.02 & -.11 & -.44 & .00 & -.05 \\
\hline 57 & -.16 & .11 & -.03 & .61 & .00 & .09 & -.09 & .09 & .04 & .60 & .07 & .07 \\
\hline 81 & -.19 & .01 & .00 & .39 & .02 & -.02 & -.13 & -.01 & .03 & .39 & .08 & -.04 \\
\hline 15 & .01 & -.05 & .05 & -.45 & -.01 & .08 & -.05 & -.03 & .03 & -.46 & -.05 & .11 \\
\hline 39 & .05 & .22 & -.20 & .44 & .05 & .10 & .08 & .20 & -.14 & .45 & .08 & .06 \\
\hline 63 & .15 & -.05 & .09 & -.35 & .14 & .06 & .11 & -.04 & .09 & -.31 & .10 & .07 \\
\hline 21 & .13 & .14 & .07 & -.50 & .34 & .03 & .00 & .14 & .08 & .53 & .30 & .04 \\
\hline 69 & -.14 & -.26 & .01 & .40 & -.22 & -.11 & -.03 & -.26 & -.03 & .45 & -.16 & -.11 \\
\hline 26 & -.07 & .11 & .00 & .15 & -.55 & .05 & .01 & .13 & -.02 & .12 & -.55 & .08 \\
\hline 74 & .20 & .02 & -.23 & .01 & .47 & -.08 & .16 & .00 & -.21 & .07 & .48 & -.13 \\
\hline 32 & -.05 & -.08 & .38 & -.05 & -.38 & -.08 & .00 & -.04 & .34 & -.04 & -.37 & -.03 \\
\hline 80 & .15 & -.04 & -.20 & .01 & .39 & .21 & .11 & -.07 & -.14 & .07 & .37 & .17 \\
\hline 38 & .13 & -.07 & -.04 & .05 & .51 & .02 & .04 & -.09 & .01 & .08 & .54 & -.02 \\
\hline 62 & -.23 & .05 & .14 & -.05 & -.60 & -.10 & -.13 & .08 & .08 & -.09 & -.59 & -.05 \\
\hline 86 & .02 & .10 & .03 & -.11 & -.48 & -.17 & .09 & .13 & -.04 & -.11 & -.50 & -.15 \\
\hline 20 & .13 & .08 & .09 & -.07 & .61 & .12 & .03 & .06 & .16 & -.05 & .61 & -.08 \\
\hline 44 & .11 & .15 & -.07 & -.18 & .63 & -.01 & .02 & .13 & -.01 & -.17 & .63 & -.05 \\
\hline 92 & .01 & -.05 & .00 & -.08 & .55 & .00 & -.04 & -.07 & .03 & -.05 & .55 & -.03 \\
\hline 1 & .02 & -.10 & .00 & -.17 & .09 & .56 & -.03 & -.11 & .06 & -.18 & .02 & .58 \\
\hline 49 & -.06 & .11 & .00 & .10 & -.12 & -.64 & .01 & .11 & -.09 & .11 & -.07 & -.67 \\
\hline 7 & .07 & -.17 & .11 & .07 & -.02 & -.44 & .08 & -.17 & .05 & .10 & .03 & -.44 \\
\hline 79 & .02 & .02 & -.01 & -.09 & .30 & .49 & -.02 & .01 & .07 & -.09 & .25 & .47 \\
\hline 37 & -.05 & .11 & .03 & .02 & -.01 & -.71 & -.01 & .11 & -.07 & .03 & .05 & -.74 \\
\hline 61 & .00 & -.03 & .40 & -.18 & .05 & -.33 & .02 & .00 & .35 & -.16 & .08 & -.33 \\
\hline 85 & -.02 & .06 & -.39 & .16 & .05 & .51 & -.05 & .04 & -.30 & .14 & .02 & .51 \\
\hline 19 & .11 & .09 & -.07 & .01 & .05 & .44 & .08 & .09 & .00 & .01 & .01 & .43 \\
\hline 43 & .02 & -.04 & .07 & -.16 & .03 & -.32 & .04 & -.03 & .01 & -.12 & .04 & -.32 \\
\hline 91 & .06 & .08 & .05 & -.01 & -.02 & .63 & .08 & .10 & .14 & .02 & -.08 & .62 \\
\hline
\end{tabular}

Note. Item numbers from the HEXACO-100. All coefficients > .30 are shown in bold. $\mathrm{H}-$ Honesty-Humility, E - Emotionality,

$\mathrm{X}$ - Extraversion, A - Agreeableness, C - Conscientiousness, O - Openness to Experience. 
Table 2 also shows standardized factor loadings from ESEM. All primary loadings and only two crossloadings (Items 32 and 61) exceeded .30. The six-factor model fitted the data well, according to RMSEA and SRMR; however, CFI was unsatisfactory. The model fit estimates were as follows: $\chi^{2}(1425)=2890.57, p<.001$, $\mathrm{CFI}=.801, \mathrm{RMSEA}=.044,90 \% \mathrm{CI}[.042, .047], p=1.000$, $\mathrm{SRMR}=.040$.

HEXACO-100. To verify the factor structure of the HEXACO-100, we first conducted ESEM on 25 facets, applying target rotation with cross-loadings specified to be close to zero. The tested six-factor model fitted the data well, $\chi^{2}(165)=389.28, p<.001, \mathrm{CFI}=.947$, $\mathrm{RMSEA}=.051,90 \% \mathrm{CI}[.044, .058], p=.388, \mathrm{SRMR}=.023$. The standardized factor loadings of the model are presented in Table 3. Expected loadings were high or moderate and all cross-loadings were low (with the highest being .38 for Diligence). Altruism was expected to be loaded by Honesty-Humility, Emotionality, and Agreeableness. The highest loading was for Emotionality. Surprisingly, the Extraversion loading was higher than loadings for Honesty-Humility and Agreeableness.

Table 3

Standardized factor loadings of the six-factor ESEM model of the HEXACO-100

\begin{tabular}{|c|c|c|c|c|c|c|}
\hline Facet & $\begin{array}{l}\text { Honesty- } \\
\text { Humility }\end{array}$ & $\begin{array}{l}\text { Emotion- } \\
\text { ality }\end{array}$ & $\begin{array}{c}\text { Extra- } \\
\text { version }\end{array}$ & $\begin{array}{l}\text { Agree- } \\
\text { ableness }\end{array}$ & $\begin{array}{l}\text { Conscien- } \\
\text { tiousness }\end{array}$ & $\begin{array}{c}\text { Openness to } \\
\text { Experience }\end{array}$ \\
\hline H_Sincerity & .56 & -.08 & -.04 & -.04 & .10 & .01 \\
\hline H_Fairness & .43 & .07 & .06 & .15 & .17 & .09 \\
\hline H_Greed Avoidance & .72 & -.09 & -.13 & .08 & -.06 & .04 \\
\hline H_Modesty & .78 & .08 & .01 & -.02 & -.05 & -.11 \\
\hline E_Fearfulness & -.10 & .63 & -.20 & .11 & .10 & -.17 \\
\hline E_Anxiety & .03 & .67 & -.24 & -.11 & .08 & .11 \\
\hline E_Dependence & -.08 & .71 & .22 & .06 & -.06 & -.05 \\
\hline E_Sentimentality & .17 & .73 & .11 & -.05 & .05 & .13 \\
\hline X_Social Self-Esteem & -.07 & -.18 & .63 & .05 & .20 & .01 \\
\hline X_Social Boldness & -.09 & -.15 & .60 & -.16 & .02 & .15 \\
\hline X_Sociability & -.08 & .27 & .76 & .14 & -.07 & -.02 \\
\hline X_Liveliness & .07 & -.10 & .75 & -.03 & .10 & -.03 \\
\hline A_Forgiveness & .09 & -.01 & .12 & .56 & -.07 & .04 \\
\hline A_Gentleness & .19 & .13 & .16 & .59 & -.06 & -.04 \\
\hline A_Flexibility & -.06 & .14 & -.10 & .67 & -.06 & .04 \\
\hline A_Patience & -.01 & -.27 & -.06 & .71 & .16 & .07 \\
\hline C_Organization & .07 & .13 & .08 & .05 & .70 & -.20 \\
\hline C_Diligence & .04 & -.03 & .38 & -.08 & .53 & .20 \\
\hline C_Perfectionism & -.01 & .17 & -.10 & -.14 & .74 & .10 \\
\hline C_Prudence & .00 & -.11 & -.10 & .14 & .75 & -.04 \\
\hline $\begin{array}{l}\text { O_Aesthetic } \\
\text { Appreciation }\end{array}$ & .07 & .20 & -.19 & .15 & .05 & .77 \\
\hline O_Inquisitiveness & -.05 & -.16 & -.06 & .12 & .12 & .57 \\
\hline O_Creativity & -.02 & .03 & .22 & -.08 & -.05 & .70 \\
\hline O_Unconventionality & -.03 & -.06 & .02 & -.06 & -.08 & .75 \\
\hline Altruism & .22 & .48 & .32 & .20 & .17 & .09 \\
\hline
\end{tabular}

Note. All coefficients $>.30$ are shown in bold. 
In Table 4 we present the results from the analysis carried out using the orthogonal non-Procrustes procedure by Barrett (2013), in which we examined the similarity of the factor structure - tested in PCA with varimax rotation - of the Polish adaptation of the HEXACO-100 to the factor structure of the original version of the inventory. We report factor loadings from both matrices and congruence coefficients calculated at facet (rows) and factor (columns) levels. Congruence coefficients, analogically to correlations, range from -1 to 1 . Coefficients higher than .85 are typically considered evidence of similarity at an acceptable level and those higher than .95 indicate very good factor replication (cf. Barrett, 1986; McCrae, Zonderman, Bond, Costa, \& Paunonen, 1996). As can be seen in Table 4, only one congruence coefficient was lower than .95 (i.e., .93 for Flexibility, which is a facet of Agreeableness). At the factor level, all coefficients were higher than .95 . The overall solution congruence was .98 , which indicates that the factor

Table 4

Target and comparison factor matrices with congruence coefficients

\begin{tabular}{|c|c|c|c|c|c|c|c|c|c|c|c|c|c|}
\hline \multirow[t]{2}{*}{ Item } & \multicolumn{6}{|c|}{ Target matrix } & \multicolumn{6}{|c|}{ Comparison matrix } & \multirow{2}{*}{$\begin{array}{c}\text { Cong } \\
\text { coef }\end{array}$} \\
\hline & $\mathrm{H}$ & $E$ & $x$ & $A$ & $\mathrm{C}$ & $\mathrm{O}$ & $\mathrm{H}$ & E & $x$ & $A$ & C & $\mathrm{O}$ & \\
\hline H_Sincerity & .78 & -.04 & -.05 & .04 & .05 & .03 & .74 & -.05 & -.03 & .01 & .14 & .03 & .99 \\
\hline H_Fairness & .68 & .17 & .10 & .17 & .27 & .02 & .58 & .12 & .07 & .26 & .23 & .11 & .97 \\
\hline H_Greed Avoidance & .75 & -.02 & -.08 & .18 & -.04 & .13 & .75 & -.02 & -.16 & .25 & .03 & .05 & .98 \\
\hline H_Modesty & .70 & .20 & -.11 & .32 & -.05 & -.06 & .77 & .17 & -.06 & .17 & .03 & -.12 & .97 \\
\hline E_Fearfulness & .01 & .64 & -.27 & .04 & .05 & -.20 & -.07 & .67 & -.32 & .14 & .09 & -.24 & .99 \\
\hline E_Anxiety & -.06 & .64 & -.42 & -.21 & .08 & .03 & .06 & .71 & -.32 & -.10 & .07 & .07 & .96 \\
\hline E_Dependence & .01 & .75 & .12 & -.04 & -.13 & -.03 & -.07 & .77 & .13 & .07 & -.10 & -.10 & .99 \\
\hline E_Sentimentality & .28 & .74 & .09 & .10 & -.02 & .09 & .19 & .78 & .04 & .02 & .06 & .11 & .98 \\
\hline X_Social Self-Esteem & .01 & -.22 & .71 & .15 & .21 & -.03 & -.07 & -.17 & .74 & .04 & .20 & .07 & .97 \\
\hline X_Social Boldness & -.09 & -.07 & .74 & -.17 & .00 & .19 & -.14 & -.14 & .71 & -.19 & -.01 & .21 & .99 \\
\hline X_Sociability & -.11 & .32 & .72 & .12 & -.12 & .02 & -.07 & .32 & .75 & .12 & -.09 & -.01 & .99 \\
\hline X_Liveliness & .05 & -.06 & .79 & .19 & .13 & .01 & .04 & -.07 & .82 & -.01 & .10 & .04 & .97 \\
\hline A_Forgiveness & .27 & .00 & .18 & .63 & -.08 & .08 & .19 & .04 & .09 & .70 & -.02 & .03 & .98 \\
\hline A_Gentleness & .22 & .09 & .00 & .76 & -.10 & .03 & .31 & .18 & .08 & .68 & .01 & -.07 & .97 \\
\hline A_Flexibility & .15 & .08 & .06 & .74 & .03 & -.01 & .02 & .17 & -.18 & .76 & .01 & .00 & .93 \\
\hline A_Patience & .03 & -.21 & .03 & .78 & .13 & .07 & .16 & -.26 & -.05 & .72 & .26 & .07 & .97 \\
\hline C_Organization & .03 & .01 & .15 & .00 & .69 & -.19 & .17 & .14 & .14 & .12 & .76 & -.14 & .95 \\
\hline C_Diligence & .06 & -.02 & .37 & -.07 & .65 & .19 & .07 & -.02 & .51 & -.04 & .56 & .31 & .98 \\
\hline C_Perfectionism & .01 & .14 & -.15 & -.12 & .69 & .15 & .06 & .16 & .00 & -.10 & .79 & .18 & .98 \\
\hline C_Prudence & .08 & -.21 & -.03 & .17 & .73 & -.02 & .14 & -.13 & .01 & .17 & .80 & .03 & .99 \\
\hline $\begin{array}{l}\text { O_Aesthetic } \\
\text { Appreciation }\end{array}$ & .17 & .15 & -.02 & .13 & .09 & .71 & .15 & .21 & -.11 & .15 & .11 & .78 & .99 \\
\hline O_Inquisitiveness & .03 & -.24 & .02 & .02 & .13 & .64 & -.03 & -.20 & .04 & .14 & .18 & .69 & .98 \\
\hline O_Creativity & .03 & .08 & .16 & .02 & -.03 & .73 & -.01 & .04 & .33 & -.13 & -.03 & .74 & .96 \\
\hline O_Unconventionality & -.04 & -.06 & .01 & .00 & -.11 & .78 & -.04 & -.06 & .13 & -.10 & -.05 & .81 & .98 \\
\hline Altruism & .46 & .47 & .20 & .37 & .08 & .15 & .32 & .56 & .30 & .27 & .20 & .10 & .96 \\
\hline Cong coef for factors & & & & & & & .97 & .99 & .97 & .96 & .98 & .99 & \\
\hline
\end{tabular}

Note. All coefficients > .30 are shown in bold. Cong coef - congruence coefficient; H - Honesty-Humility; E - Emotionality; $\mathrm{X}$ - Extraversion; A - Agreeableness; C - Conscientiousness; O - Openness to Experience. 
structure of the Polish adaptation of the HEXACO-100 is a very good replication of the original version of the inventory.

In general, the results presented above confirm the factor validity of the Polish adaptations of the HEXACO-60 and HEXACO-100 (H3).

\section{CORRELATIONS AMONG THE HEXACO FACTOR SCALES (H4)}

Table 5 shows correlations among the HEXACO factor scales: separately for the HEXACO-60 and the HEXACO-100 inventories. In both inventories there was one moderate correlation coefficient: between Honesty-Humility and Agreeableness. Similarly, in validation studies of the original version of the HEXACO-100, the correlation between these two factors exceeded .40 (Lee \& Ashton, 2018). All other correlations were low $(<.30)$, as expected (confirming $\mathrm{H} 4$ ).

\section{CORRELATIONS WITH OTHER PERSONALITY MEASURES (H5-H9)}

Correlations with QB6 scales. We correlated HEXACO-60 and HEXACO-100 basic scales with measures of the Big Six and Big Two personality factors included in the QB6. Correlation coefficients are presented in Table 6.

As can be seen in Table 6, each HEXACO scale showed the highest correlation with its Big Six counterpart, confirming H5. However, some HEXACO scales also correlated moderately with other Big Six scales: Honesty-Humility with Agreeableness, Extraversion with Resiliency and Originality, and Conscientiousness with Honesty/Propriety and Originality.

Correlations with the Big Two scales were as expected (H8 and H9): Social Self-Regulation correlated moderately with Honesty-Humility, Agreeableness, and Conscientiousness; Dynamism correlated highly with Extraversion and moderately with

Table 5

Correlations among the HEXACO-60 (above the diagonal) and HEXACO-100 (below the diagonal) factor scales

\begin{tabular}{lcccccc}
\hline HEXACO scale & $\begin{array}{c}\text { Honesty- } \\
\text { Humility }\end{array}$ & $\begin{array}{c}\text { Emotion- } \\
\text { ality }\end{array}$ & $\begin{array}{c}\text { Extra- } \\
\text { version }\end{array}$ & $\begin{array}{c}\text { Agree- } \\
\text { ableness }\end{array}$ & $\begin{array}{c}\text { Conscien- } \\
\text { tiousness }\end{array}$ & $\begin{array}{c}\text { Openness to } \\
\text { Experience }\end{array}$ \\
\hline Honesty-Humility & .12 & -.09 & .42 & .28 & .09 \\
Emotionality & .14 & & -.20 & .11 & .03 & -.05 \\
Extraversion & -.11 & -.16 & & -.05 & .22 & .21 \\
Agreeableness & .44 & .09 & -.06 & & .16 & .03 \\
Conscientiousness & .28 & .05 & .24 & .18 & & .19 \\
Openness to Experience & .08 & -.07 & .22 & .05 & .21 & \\
\hline
\end{tabular}

Note. $N=522$. All coefficients $>|.09|$ are significant at $p<.05$. All coefficients $>|.11|$ are significant at $p<.01$. All coefficients $>|.14|$ are significant at $p<.001$.

Table 6

Correlations of the HEXACO-60/HEXACO-100 with the Big Six and Big Two scales from the QB6

\begin{tabular}{lcccccccc}
\hline HEXACO scale & $\begin{array}{c}\text { Honesty/ } \\
\text { Propriety }\end{array}$ & $\begin{array}{c}\text { Resil- } \\
\text { iency }\end{array}$ & $\begin{array}{c}\text { Extra- } \\
\text { version }\end{array}$ & $\begin{array}{c}\text { Agree- } \\
\text { ableness }\end{array}$ & $\begin{array}{c}\text { Conscien- } \\
\text { tiousness }\end{array}$ & $\begin{array}{c}\text { Origi- } \\
\text { nality }\end{array}$ & $\begin{array}{c}\text { Social Self- } \\
\text { Regulation }\end{array}$ & $\begin{array}{c}\text { Dyna- } \\
\text { mism }\end{array}$ \\
\hline Honesty-Humility & $.43 / .42$ & $-.01 /-.03$ & $-.09 /-.10$ & $.41 / .41$ & $.24 / .23$ & $-.06 /-.09$ & $.44 / .43$ & $-.13 /-.17$ \\
Emotionality & $.20 / .21$ & $-.61 /-.61$ & $.08 / .08$ & $-.13 /-.14$ & $.07 / .07$ & $-.17 /-.17$ & $.01 / .02$ & $-.23 /-.22$ \\
Extraversion & $.00 / .02$ & $.39 / .39$ & $.60 / .65$ & $.01 / .02$ & $.30 / .30$ & $.36 / .36$ & $.14 / .16$ & $.62 / .64$ \\
Agreeableness & $.29 / .25$ & $.02 / .03$ & $-.05 /-.06$ & $.57 / .58$ & $.20 / .18$ & $-.13 /-.13$ & $.44 / .41$ & $-.17 /-.19$ \\
Conscientiousness & $.32 / .33$ & $.11 / .14$ & $.09 / .11$ & $.25 / .28$ & $.61 / .68$ & $.32 / .31$ & $.52 / .55$ & $.20 / .21$ \\
Openness to & $.03 / .03$ & $.07 / .09$ & $.09 / .10$ & $.08 / .07$ & $.02 / .04$ & $.43 / .43$ & $.08 / .09$ & $.29 / .31$ \\
Experience & & & & & & & & \\
\hline
\end{tabular}

Note. $N=518$. All coefficients $>|.09|$ are significant at $p<.05$. All coefficients $>|.11|$ are significant at $p<.01$. All coefficients $>|.14|$ are significant at $p<.001$. 
Openness to Experience. Only the negative correlation between Dynamism and Emotionality was lower than expected.

Correlations with the Big Five measures. The HEXACO-60 and the HEXACO-100 were also correlated with two popular measures of the Big Five. Correlation coefficients with the BFAS scales are presented in Table 7 and correlations with the BFI scales are presented in Table 8.

We expected the strongest relationships between HEXACO Extraversion, Conscientiousness, and Openness to Experience and their Big Five counterparts (H6). This hypothesis was confirmed by the data. The strongest correlations were between Extraversion measured by the HEXACO inventories and Extraversion measured by the BFAS and the BFI. Correlations between measures of Openness to Experience and Conscientiousness from HEXACO and the Big Five models exceeded .60.

Honesty-Humility correlated moderately with Agreeableness (slightly higher with BFAS than with the BFI Agreeableness scale). Emotionality was only moderately related with Neuroticism when the Big Five was measured by the BFI, but when it was measured by the BFAS, Emotionality also correlated mod- erately with Agreeableness. HEXACO Agreeableness was more strongly related to its BFI than to its BFAS counterpart.

We expected that Big Five Agreeableness would correlate similarly with three HEXACO scales: Honesty-Humility, Emotionality, and Agreeableness (H7). This hypothesis was confirmed when measuring Agreeableness with the BFAS: The correlation with Agreeableness was even lower than with Honesty-Humility and Emotionality.

Correlations with the Big Five Aspects. Table 9 presents correlations between the Big Five aspects measured by the BFAS and the HEXACO domains and facets. Honesty-Humility and its facets are most strongly related to Politeness, which is in line with previous findings (Barford, Zhao, \& Smillie, 2015; Ludeke et al., 2019).

Emotionality was substantially related to both aspects of Neuroticism (Volatility and Withdrawal) and Compassion, with the highest correlation with Withdrawal. This is also consistent with previous observations (Ludeke et al., 2019). However, in this study, the correlations with Compassion and Volatility were slightly higher than those found by Ludeke et al. (2019), which were .38 and .40 , respectively.

Table 7

Correlations between the HEXACO-60/HEXACO-100 and the BFAS scales

\begin{tabular}{lccccc}
\hline HEXACO scale & Neuroticism & Agreeableness & Conscientiousness & Extraversion & Openness \\
\hline Honesty-Humility & $-.08 /-.06$ & $.43 / .46$ & $.23 / .22$ & $-.14 /-.17$ & $.15 / .13$ \\
Emotionality & $.56 / .56$ & $.41 / .42$ & $-.02 /-.02$ & $-.14 /-.11$ & $.03 / .05$ \\
Extraversion & $-.36 /-.36$ & $-.01 / .03$ & $.26 / .25$ & $.71 / .73$ & $.25 / .26$ \\
Agreeableness & $-.22 /-.23$ & $.35 / .34$ & $.17 / .16$ & $-.15 /-.16$ & $-.02 /-.01$ \\
Conscientiousness & $-.23 /-.27$ & $.22 / .23$ & $.58 / .63$ & $.05 / .08$ & $.22 / .22$ \\
Openness to Experience & $-.08 /-.10$ & $.11 / .10$ & $.04 / .04$ & $.24 / .25$ & $.69 / .69$ \\
\hline
\end{tabular}

Note. $N=504$. All coefficients $>|.09|$ are significant at $p<.05$. All coefficients $>|.11|$ are significant at $p<.01$. All coefficients $>|.15|$ are significant at $p<.001$.

Table 8

Correlations between the HEXACO-60/HEXACO-100 and the BFI scales

\begin{tabular}{lccccc}
\hline HEXACO scale & Neuroticism & Agreeableness & Conscientiousness & Extraversion & Openness \\
\hline Honesty-Humility & $-.09 /-.07$ & $.39 / .39$ & $.23 / .22$ & $-.15 /-.18$ & $.00 /-.03$ \\
Emotionality & $.52 / .52$ & $.22 / .24$ & $.01 / .02$ & $-.09 /-.07$ & $-.01 / .00$ \\
Extraversion & $-.40 /-.40$ & $.18 / .21$ & $.24 / .23$ & $.67 / .70$ & $.31 / .32$ \\
Agreeableness & $-.20 /-.21$ & $.53 / .53$ & $.18 / .15$ & $-.13 /-.14$ & $-.11 /-.12$ \\
Conscientiousness & $-.19 /-.23$ & $.16 / .21$ & $.62 / .66$ & $.00 / .02$ & $.08 / .09$ \\
Openness to Experience & $-.14 /-.14$ & $.11 / .10$ & $.08 / .08$ & $.14 / .15$ & $.64 / .64$ \\
\hline
\end{tabular}

Note. $N=505$. All coefficients $>|.09|$ are significant at $p<.05$. All coefficients $>|.11|$ are significant at $p<.01$. All coefficients $>|.15|$ are significant at $p<.001$. 
Table 9

Correlations between the HEXACO domains and facets and the Big Five aspects

\begin{tabular}{|c|c|c|c|c|c|c|c|c|c|c|}
\hline \multirow[t]{2}{*}{ HEXACO facet } & \multicolumn{2}{|c|}{ Neuroticism } & \multicolumn{2}{|c|}{$\begin{array}{l}\text { Agreeable- } \\
\text { ness }\end{array}$} & \multicolumn{2}{|c|}{$\begin{array}{l}\text { Conscien- } \\
\text { tiousness }\end{array}$} & \multicolumn{2}{|c|}{ Extraversion } & \multicolumn{2}{|c|}{ Openness } \\
\hline & Nv & $\mathrm{Nw}$ & Ac & Ap & $\mathrm{Ci}$ & Co & $\mathrm{Ee}$ & $\mathrm{Ea}$ & Oi & Oo \\
\hline $\begin{array}{l}\text { Honesty- } \\
\text { Humility-60/100 }\end{array}$ & $-.12 /-.11$ & $-.02 / .00$ & $.33 / .33$ & $.44 / .47$ & $.18 / .16$ & $.21 / .21$ & $.00 /-.00$ & $-.20 /-.25$ & $.03 / .00$ & $.20 / .20$ \\
\hline H_Sincerity & -.13 & -.04 & .18 & .28 & .15 & .16 & -.03 & -.09 & .04 & .10 \\
\hline H_Fairness & -.12 & -.06 & .30 & .36 & .18 & .20 & .05 & -.09 & .07 & .22 \\
\hline H_Greed Avoidance & -.06 & .03 & .20 & .35 & .10 & .09 & -.10 & -.25 & -.01 & .16 \\
\hline H_Modesty & .00 & .10 & .33 & .44 & .04 & .19 & .04 & -.32 & -.10 & .08 \\
\hline Emotionality-60/100 & $.47 / .47$ & $.56 / .56$ & $.46 / .48$ & $.23 / .23$ & $-.19 /-.18$ & $3.17 / .16$ & $.13 / .15$ & $-.32 /-.30$ & $-.22 /-.20$ & $.24 / .26$ \\
\hline E_Fearfulness & .33 & .42 & .24 & .17 & -.17 & .16 & -.02 & -.35 & -.29 & .03 \\
\hline E_Anxiety & .39 & .55 & .36 & .17 & -.21 & .11 & -.03 & -.30 & -.12 & .27 \\
\hline E_Dependence & .35 & .37 & .35 & .13 & -.13 & .06 & .28 & -.12 & -.15 & .17 \\
\hline E_Sentimentality & .37 & .39 & .55 & .25 & -.04 & .14 & .21 & -.15 & -.04 & .34 \\
\hline Extraversion-60/100 & $-.19 /-.20$ & $-.48 /-.47$ & $.07 / .11$ & $-.10 /-.07$ & $7.40 / .39$ & $.03 / .03$ & $.52 / .58$ & $.64 / .61$ & $.39 / .39$ & $.04 / .06$ \\
\hline X_Social Self-Esteem & -.28 & -.53 & .01 & -.02 & .42 & .10 & .37 & .49 & .33 & .00 \\
\hline X_Social Boldness & -.09 & -.35 & -.04 & -.25 & .25 & -.07 & .34 & .66 & .42 & .09 \\
\hline X_Sociability & -.02 & -.12 & .27 & .03 & .14 & -.04 & .53 & .26 & .14 & .06 \\
\hline X_Liveliness & -.23 & -.46 & .13 & .03 & .40 & .09 & .57 & .49 & .30 & .02 \\
\hline Agreeableness-60/100 & $-.31 /-.32$ & $-.08 /-.08$ & $.22 / .21$ & $.40 / .40$ & $.13 / .12$ & $.17 / .15$ & $.06 / .05$ & $-.28 /-.29$ & $-.11 /-.09$ & $.07 / .07$ \\
\hline A_Forgiveness & -.18 & -.08 & .16 & .22 & .05 & .08 & .14 & -.12 & -.01 & .09 \\
\hline A_Gentleness & -.13 & .02 & .28 & .39 & .04 & .15 & .12 & -.28 & -.15 & .04 \\
\hline A_Flexibility & -.10 & .08 & .15 & .31 & .06 & .11 & -.04 & -.32 & -.14 & .05 \\
\hline A_Patience & -.55 & -.25 & .05 & .31 & .20 & .13 & -.07 & -.17 & .01 & .01 \\
\hline $\begin{array}{l}\text { Conscientiousness- } \\
60 / 100\end{array}$ & $-.22 /-.26$ & $-.20 /-.24$ & $.17 / .17$ & $.22 / .24$ & $.50 / .54$ & $.48 / .53$ & $.00 / .03$ & $.08 / .09$ & $.30 / .30$ & $.08 / .08$ \\
\hline C_Organization & -.16 & -.15 & .15 & .26 & .46 & .60 & .03 & -.04 & .11 & .02 \\
\hline C_Diligence & -.23 & -.34 & .12 & .05 & .48 & .24 & .20 & .35 & .44 & .13 \\
\hline C_Perfectionism & -.06 & -.02 & .20 & .20 & .33 & .42 & -.01 & .01 & .20 & .13 \\
\hline C_Prudence & -.35 & -.23 & .05 & .21 & .42 & .37 & -.13 & .00 & .19 & -.03 \\
\hline $\begin{array}{l}\text { Openness to } \\
\text { Experience-60/100 }\end{array}$ & $-.04 /-.05$ & $-.11 /-.13$ & $.20 / .18$ & $-.02 /-.02$ & $2.10 / .11$ & $-.04 /-.04$ & $4.12 / .12$ & $.26 / .28$ & $.52 / .52$ & $.62 / .62$ \\
\hline $\begin{array}{l}\text { O_Aesthetic } \\
\text { Appreciation }\end{array}$ & .02 & -.05 & .29 & .15 & .06 & .07 & .01 & -.01 & .25 & .62 \\
\hline O_Inquisitiveness & -.11 & -.19 & .02 & -.07 & .12 & .01 & -.01 & .15 & .39 & .29 \\
\hline O_Creativity & -.04 & -.17 & .16 & -.05 & .12 & -.10 & .24 & .44 & .46 & .51 \\
\hline O_Unconventionality & -.02 & -.10 & .07 & -.13 & .02 & -.15 & .15 & .34 & .50 & .47 \\
\hline Altruism & .08 & .15 & .52 & .41 & .11 & .25 & .29 & -.12 & .03 & .26 \\
\hline
\end{tabular}

Note. $N=504 . \mathrm{Nv}$ - Volatility, Nw - Withdrawal, Ac - Compassion, Ap - Politeness, Ci - Industriousness, Co - Orderliness, Ee - Enthusiasm, Ea - Assertiveness, Oi - Intellect, Oo - Openness to Experience. All coefficients $>|.09|$ are significant at $p<.05$. All coefficients $>|.11|$ are significant at $p<.01$. All coefficients $>|.15|$ are significant at $p<.001$. 
Sentimentality was the facet most strongly related to Compassion $(r=.55)$.

The patterns of correlations found in this study for Extraversion, Openness to Experience and Altruism were identical to those from the study by Ludeke et al. (2019). Extraversion was strongly related to Assertiveness and Enthusiasm, but also moderately to Withdrawal (negatively), Industriousness, and Intellect. Openness to Experience was only strongly related to Openness and Intellect. Altruism was primarily correlated with both aspects of Agreeableness and secondarily with Enthusiasm.

There were some small differences between the results of this study and the results obtained by Ludeke et al. (2019) regarding Agreeableness and Conscientiousness. In this study, Agreeableness was primarily associated with Politeness and secondarily with Volatility (negatively), whereas Ludeke et al. found a higher correlation with Volatility than with Politeness. Conscientiousness was strongly related to Industriousness and Orderliness and moderately to Intellect in both studies. However, in Ludeke et al.'s study it was also moderately associated with Assertiveness, whereas in this study we found a correlation with this aspect for only one Conscientiousness facet, which was Diligence.

\section{DISCUSSION}

The findings from this study confirmed that the Polish adaptations of the HEXACO-60 and the HEXACO- 100 inventories are reliable and valid measures of the Big Six personality dimensions. The internal consistencies of the scales do not differ from their original versions. Additionally, the omega general coefficients showed that the general factor saturation of the six basic scales (which comprise four facet factors each) is higher than that found by Wilt and Revelle (2019) for the five basic scales (they used Big Five scales representing four components of each trait: affect, behavior, cognition, and desire). This may suggest that the five basic factors of personality traits are more internally diversified and the six basic factors are more consistent. This requires further examination of data collected with different measures of these two competing models of basic personality traits.

Internal consistency reliability coefficients for the six basic scales measured by the HEXACO-60 were only slightly lower than those for the basic scales measured by the HEXACO-100. Hence, we recommend using this shortened version when the aim of a study is to measure only the six basic HEXACO dimensions. A researcher interested in measuring the HEXACO facets is encouraged to use the HEXACO-100. Most of the facet scales had satisfactory $\alpha$ and $\omega$ coefficients. Only for three of them were the coefficients lower than .60 (although not lower than .55). These were:
Anxiety (Emotionality), Unconventionality (Openness to Experience), and Altruism (interstitial scale). Cronbach's $\alpha$ coefficients below .60 for some of the facet scales also occurred in the original version of the HEXACO-100 (Lee \& Ashton, 2018) and in other language versions, for instance Spanish (Romero, Villar, \& López-Romero, 2015) or, especially, Lithuanian (Truskauskaitè-Kunevičienè, Kaniušonyte, Kratavičien, \& Kratavičiūtè-Ališauskienè, 2012). Lower internal consistency is replicable in different languages especially for Altruism and Unconventionality. When interpreting $\alpha$ and $\omega$ coefficients for facet scales of the HEXACO-100, one should take into account that they consist of only four items each. A more reliable measure of the HEXACO facets is available with the longer scales of the HEXACO-200.

Previous studies typically verified the factor structure of other language adaptations of instruments measuring the HEXACO model in exploratory analyses (such as principal component analysis or principal axis extraction). However, their results should not be interpreted in terms of confirmation. In this study, we used exploratory structural equation modeling (ESEM), which allowed us to confirm the six-factor structure of the HEXACO-60 and the HEXACO-100, revealing high or moderate expected loadings and low cross-loadings.

The use of an exploratory factor comparison analysis was another methodological novelty of this study in comparison with other studies that examined psychometric properties of language adaptations of the HEXACO inventories. High congruence coefficients showed that the Polish version of the HEXACO-100 is a very good replication of the original inventory.

However, we noted one deviation in intercorrelations of the HEXACO scales. Honesty-Humility correlated moderately with Agreeableness - not only measured by the same inventory, but also with its counterparts from the Big Five and Big Six models. Its correlation with the Agreeableness scale from the QB6 was almost identical to the correlation with the corresponding Honesty-Propriety scale. The higher than expected correlation between HonestyHumility and Agreeableness is most likely caused by strong prosocial content (or even core) present in both factors and it also showed up in the study on a community sample that used the original version of the HEXACO-100 (Lee \& Ashton, 2018). It did not replicate in other language adaptations, however (Međedović, Čolović, Dinić, \& Smederevac, 2019; Romero et al., 2015; Truskauskaitè-Kunevičienė et al., 2012). Despite this fact, the distinctiveness of the Honesty-Humility factor should not be questioned because of the lack of cross-loadings in both variants of the conducted factor analysis: PCA and ESEM.

The correlation analyses of the HEXACO basic scales and the lexical Big Six factors measured by the QB6 showed the highest similarity between 
the dimensions of Extraversion, Emotionality (vs. Resiliency), and Conscientiousness. Honesty-Propriety was moderately related not only to HonestyHumility, but also to Conscientiousness. HEXACO Agreeableness only correlated moderately with QB6 Agreeableness, but the latter was also moderately related with HEXACO Honesty-Humility. Openness to Experience only correlated moderately with Originality, but the latter was also moderately related with HEXACO Extraversion and Conscientiousness. Overall, the pattern of correlations between the HEXACO and the Big Six indicates a considerable similarity of the two measures of the very similar, albeit not identical models. Each HEXACO scale showed the highest correlation with its QB6 counterpart and correlations with other scales reflected intercorrelations among HEXACO domains, as presented in Table 5.

Regarding the relations of the HEXACO basic scales with the lexical Big Two measured by the QB6, Social-Self Regulation correlated positively with Honesty-Humility, Agreeableness, and Conscientiousness and did not correlate with other HEXACO scales, as expected (see Strus \& Cieciuch, 2019). Dynamism positively correlated with Extraversion and moderately with Openness to Experience. At a similar level, Dynamism also correlated with Emotionality (negatively) and with Conscientiousness (positively). Based on the definition of Dynamism, it should be particularly negatively related to Fearfulness, which is a facet of Emotionality. For this reason, a negative relationship between Dynamism and Emotionality was expected. The correlation coefficient between the two variables in this study was lower than |.30|, but higher than that from the study conducted by Saucier et al. (2014). The lower than expected correlation between Dynamism and Emotionality in these two studies may indicate that the emotional aspect of Dynamism is dominated by other aspects of this broad trait (especially related to Extraversion).

The patterns of correlations between the HEXACO and Big Five basic scales might serve as further evidence for the Polish versions of the HEXACO-60 and the HEXACO-100 being valid operationalizations of the HEXACO model of personality. The highest correlations were found between Extraversion, Conscientiousness, and Openness to Experience measured by the HEXACO inventories and Extraversion, Conscientiousness, and Openness measured by the BFAS and the BFI, which was expected, as these three HEXACO basic traits are the most similar to their Big Five counterparts. When Big Five traits were measured by the BFAS, the results showed a stronger alignment with expectations. BFAS Agreeableness was related similarly to three HEXACO factors, i.e. Agreeableness, Honesty-Humility, and Emotionality, showing that the two Agreeableness factors - from the HEXACO and from the Big Five models - con- ceptually differ from each other. The same pattern of correlations was found by Ludeke et al. (2019) for the BFAS Agreeableness scale, but not by Ashton et al. (2019) for the BFI Agreeableness scale, which did not correlate with Emotionality.

The pattern of correlations between the HEXACO domains and the Big Five aspects was largely consistent with results obtained by Ludeke et al. (2019) in a meta-analysis of four samples. This further confirms the validity of the Polish adaptations of the HEXACO-60 and the HEXACO-100 inventories. What is more, a moderate correlation between Honesty-Humility and Politeness shows that these two traits, although associated, are conceptually distinct. This finding undermines DeYoung's (2015) claim that Honesty-Humility reflects only one aspect of Agreeableness (i.e., Politeness) instead of constituting a dimension that adds to the FFM (see Ludeke et al., 2019).

To conclude, we find the psychometric properties of the Polish versions of the HEXACO-60 and the HEXACO-100 inventories satisfactory and recommend using them in research. Both inventories are reliable and valid measures of the six basic dimensions distinguished in the HEXACO model of personality traits. We believe that the HEXACO model can be useful to predict various outcomes, especially related to moral functioning, due to the inclusion of the additional dimension of Honesty-Humility.

\section{FUNDING}

The work of ES was supported by Grant 2018/28/T/ HS6/00224 from the National Science Centre, Poland. The work of WS was supported by Grant 2014/14/M/ HS6/00919 from the National Science Centre, Poland. The work of JC was supported by the University Research Priority Program Social Networks of the University of Zurich.

\section{ENDNOTE}

1 Psychometric properties of the original version of the HEXACO-100 were examined with much larger samples of online respondents $(N=100,318)$ and undergraduate students $(N=2,868$; Lee \& Ashton, 2018). Psychometric properties of the original version of the HEXACO-60 were examined with a sample of students $(N=936)$ and a community sample $(N=734$; Ashton \& Lee, 2009). Psychometric properties of other language adaptations of the inventories were examined with various samples - often of a similar size or smaller and less representative than the community sample in this study (e.g., de Vries et al., 2008; TruskauskaitéKunevičienè et al., 2012; Wakabayashi, 2014). 


\section{References}

Aghababaei, N. (2012). Religious, honest and humble: Looking for the religious person within the HEXACO model of personality structure. Personality and Individual Differences, 53, 880-883. https:// doi.org/10.1016/j.paid.2012.07.005

Ashton, M. C., \& Lee, K. (2001). A theoretical basis for the major dimensions of personality. European Journal of Personality, 15, 327-353. https://doi. org/10.1002/per.417

Ashton, M. C., \& Lee, K. (2007). Empirical, theoretical, and practical advantages of the HEXACO model of personality structure. Personality and Social Psychology Review, 11, 150-166. https://doi. org/10.1177/1088868306294907

Ashton, M. C., \& Lee, K. (2008). The HEXACO model of personality structure. In G. J. Boyle, G. Matthews, \& D. Saklofske (Eds.), Handbook of personality theory and testing. Vol. 2: Personality measurement and assessment (pp. 239-260). London: Sage.

Ashton, M. C., \& Lee, K. (2009). The HEXACO-60: a short measure of the major dimensions of personality. Journal of Personality Assessment, 91, 340345. https://doi.org/10.1080/00223890902935878

Ashton, M. C., \& Lee, K. (2018). How well do Big Five measures capture HEXACO scale variance? Journal of Personality Assessment, 9, 1-7. https://doi.org/10. 1080/00223891.2018.1448986

Ashton, M. C., \& Lee, K. (2019). Religiousness and the HEXACO personality factors and facets in a large online sample. Journal of Personality, 87, 1103-1118. https://doi.org/10.1111/jopy. 12459

Ashton, M. C., Lee, K., Marcus, B., \& de Vries, R. E. (2007). German lexical personality factors: Relations with the HEXACO model. European Journal of Personality, 21, 23-43. https://doi.org/10.1002/ per.597

Ashton, M. C., Lee, K., Perugini, M., Szarota, P., de Vries, R. E., Di Blas, L., Boies, K., \& De Raad, B. (2004). A six-factor structure of personality-descriptive adjectives: Solutions from psycholexical studies in seven languages. Journal of Personality and Social Psychology, 86, 356-366. https://doi. org/10.1037/0022-3514.86.2.356

Ashton, M. C., Lee, K., Pozzebon, J. A., Visser, B. A., \& Worth. N. C. (2010). Status-driven risk taking and the major dimensions of personality. Journal of Research in Personality, 44, 734-737. https://doi. org/10.1016/j.jrp.2010.09.003

Ashton, M. C., Lee, K., \& Visser, B. A. (2019). Where's the H? Relations between BFI-2 and HEXACO-60 scales. Personality and Individual Differences, 137, 71-75. https://doi.org/10.1016/j.paid.2018.08.013

Asparouhov, T., \& Muthén, B. (2009). Exploratory structural equation modeling. Structural Equation Modeling: A Multidisciplinary Journal, 16, 397-438. https://doi.org/10.1080/10705510903008204
Barford, K. A., Zhao, K., \& Smillie, L. D. (2015). Mapping the interpersonal domain: Translating between the Big Five, HEXACO, and Interpersonal Circumplex. Personality and Individual Differences, 86, 232-237. https://doi.org/10.1016/j.paid.2015.05.038

Barrett, P. (1986). Factor comparison: An examination of three methods. Personality and Individual Differences, 7, 327-340. https://doi.org/10.1016/01918869(86)90008-5

Barrett, P. (2013). Orthosim target-comparison matrix fitting. Retrieved from https://www.pbarrett.net/

Becker, L. A. (1998). Effect Size Calculators [computer software]. Retrieved from https://www.uccs. edu/ lbecker/

Browne, M. W. (2001). An overview of analytic rotation in exploratory factor analysis. Multivariate Behavioral Research, 36, 111-150. https://doi. org/10.1207/S15327906MBR3601_05

Cohen, T. R., Wolf, S. T., Panter, A. T., \& Insko, C. A. (2011). Introducing the GASP scale: a new measure of guilt and shame proneness. Journal of Personality and Social Psychology, 100, 947-966. https://doi.org/10.1037/a0022641

Condon, D., \& Revelle, W. (2014). The international cognitive ability resource: Development and initial validation of a public-domain measure. Intelligence, 43, 52-64. https://doi.org/10.1016/j.intell.2014.01.004

De Raad, B. (2009). Structural models of personality. In P. J. Corr \& G. Matthews (Eds.), The Cambridge handbook of personality psychology (pp. 127-147). New York, NY: Cambridge University Press.

De Raad, B., Barelds, D. P. H., Levert, E., Ostendorf, F., Mlacic, B., Di Blas, L., Hřebíčková, M., Szirmák, Z., Szarota, P., Perugini, M., Church, A. T., \& Katigbak, M. S. (2010). Only three factors of personality description are fully replicable across languages: a comparison of 14 trait taxonomies. Journal of Personality and Social Psychology, 98, 160-173. https://doi.org/10.1037/a0017184

de Vries, R. E., de Vries, A., de Hoogh, A., \& Feij, J. (2009). More than the Big Five: Egoism and the HEXACO model of personality. European Journal of Personality, 23, 635-654. https://doi.org/10.1002/ per.733

de Vries, R. E., Lee, K., \& Ashton, M. C. (2008). The Dutch HEXACO personality inventory: Psychometric properties, self-other agreement, and relations with psychopathy among low and high acquaintanceship dyads. Journal of Personality Assessment, 90, 142-151. https://doi.org/10.1080/00223890 701845195

DeYoung, C. G. (2015). Cybernetic Big Five Theory. Journal of Research in Personality, 56, 33-58. https:// doi.org/10.1016/j.jrp.2014.07.004

DeYoung, C. G., Quilty, L. C., \& Peterson, J. B. (2007). Between facets and domains: 10 aspects of the Big Five. Journal of Personality and Social Psychology, 93, 888-896. https://doi.org/10.1037/0022-3514.93.5.880 
Goldberg, L. R. (1990). An alternative "description of personality": The Big-Five factor structure. Journal of Personality and Social Psychology, 59, 12161229. https://doi.org/10.1037/0022-3514.59.6.1216

Goldberg, L. R. (1999). A broad-bandwidth, publicdomain, personality inventory measuring the lower-level facets of several Five-Factor models. In I. Mervielde, I. Deary, F. De Fruyt, \& F. Ostendorf (Eds.), Personality psychology in Europe (Vol. 7, pp. 7-28). Tilburg: Tilburg University Press.

Goldberg, L. R., Johnson, J. A., Eber, H. W., Hogan, R., Ashton, M. C., Clonninger, C. R., \& Gough, H. G. (2006). The International Personality Item Pool and the future of public-domain personality measures. Journal of Research in Personality, 40, 84-96. https://doi.org/10.1016/j.jrp.2005.08.007

Gorbaniuk, O., Budzińska, A., Owczarek, M., Bożek, E., \& Juros, K. (2013). The factor structure of Polish personality-descriptive adjectives: an alternative psycho-lexical study. European Journal of Personality, 27, 304-318. https://doi.org/10.1002/ per. 1921

Hilbig, B. E., \& Zettler, I. (2015). When the cat's away, some mice will play: a basic trait account of dishonest behavior. Journal of Research in Personality, 57, 72-88. https://doi.org/10.1016/j.jrp.2015.04.003

Hilbig, B. E., Zettler, O., Leist, F., \& Heydasch, T. (2013). It takes two: Honesty-Humility and Agreeableness differentially predict active versus reactive cooperation. Personality and Individual Differences, 54, 598-603. https://doi.org/10.1016/j.paid.2012.11.008

Hopwood, C. J., \& Donnellan, M. B. (2010). How should the internal structure of personality inventories be evaluated? Personality and Social Psychology Review, 14, 332-346. https://doi.org/10. 1177/1088868310361240

John, O. P., Donahue, E. M., \& Kentle, R. L. (1991). The Big Five Inventory - Versions $4 a$ and 54. Berkeley, CA: Institute of Personality and Social Research, University of California.

John, O. P., Naumann, L. P., \& Soto, Ch. J. (2008). Paradigm shift to the integrative Big Five trait taxonomy: History, measurement, and conceptual issues. In O. P. John, R. W. Robins, \& L. A. Pervin (Eds.), Handbook of personality: Theory and research (pp. 114-158). New York, NY: Guilford Press.

Lee, K., \& Ashton, M. C. (2004). Psychometric properties of the HEXACO Personality Inventory. Multivariate Behavioral Research, 39, 329-358. https:// doi.org/10.1207/s15327906mbr3902_8

Lee, K., \& Ashton, M. C. (2005). Psychopathy, Machiavellianism, and Narcissism in the Five-Factor Model and the HEXACO model of personality structure. Personality and Individual Differences, 38, 15711582. https://doi.org/10.1016/j.paid.2004.09.016

Lee, K., \& Ashton, M. C. (2008). The HEXACO personality factors in the indigenous personality lexicons of English and 11 other languages. Journal of
Personality, 76, 1002-1054. https://doi.org/10.1111/ j.1467-6494.2008.00512.x

Lee, K., \& Ashton, M. C. (2009). Reanalysis of the structure of the Greek personality lexicon. Journal of Cross-Cultural Psychology, 40, 693-700. https:// doi.org/10.1177/0022022109335183

Lee, K., \& Ashton, M. C. (2012). Getting mad and getting even: Agreeableness and Honesty-Humility as predictors of revenge intentions. Personality and Individual Differences, 52, 596-600. https://doi. org/10.1016/j.paid.2011.12.004

Lee, K., \& Ashton, M. C. (2013). Prediction of self- and observer report scores on HEXACO-60 and NEOFFI scales. Journal of Research in Personality, 47, 668-675. https://doi.org/10.1016/j.jrp.2013.06.002

Lee, K., \& Ashton, M. C. (2018). Psychometric properties of the HEXACO-100. Assessment, 25, 543-556. https://doi.org/10.1177/1073191116659134

Ludeke, S. G., Bainbridge, T. F., Liu, J., Zhao, K., Smillie, L. D., \& Zettler, I. (2019). Using the Big Five Aspect Scales to translate between the HEXACO and Big Five personality models. Journal of Personality. Advance online publication. https://doi. org/10.1111/jopy. 12453

McCrae, R. R. (1990). Traits and trait names: How well is Openness represented in natural languages? European Journal of Personality, 4, 119-129. https:// doi.org/10.1002/per.2410040205

McCrae, R. R., \& Costa, P. T., Jr. (2003). Personality in adulthood: a Five-Factor Theory perspective (2nd ed.). New York, NY: Guilford.

McCrae, R. R., Zonderman, A. B., Bond, M. H., Costa, P. T., \& Paunonen, S. V. (1996). Evaluating replicability of factors in the Revised NEO Personality Inventory: Confirmatory factor analysis versus Procrustes rotation. Journal of Personality and Social Psychology, 70, 552-566. https://doi. org/10.1037/0022-3514.70.3.552

McDonald, R. P. (1978). Generalizability in factorable domains: "Domain validity and generalizability”. Educational and Psychological Measurement, 38, 75-79. https://doi.org/10.1177/001316447803800111

McDonald, R. P. (1999). Test theory: a unified treatment. Mahwah, NJ: L. Erlbaum Associates.

McGrath, D. S., Neilson, T., Lee, K., Rash, Ch. L., \& Rad, M. (2018). Associations between the HEXACO model of personality and gambling involvement, motivations to gamble, and gambling severity in young adult gamblers. Journal of Behavioral Addictions, 7, 392-400. https://doi.org/10.1556/2006.7.2018.29

Međedović, J., Čolović, P., Dinić, B. M., \& Smederevac, S. (2019). The HEXACO Personality Inventory: Validation and psychometric proprieties in the Serbian Language. Journal of Personality Assessment, 101, 25-31. https://doi.org/10.1080/00223891. 2017.1370426

Peabody, D., \& De Raad, B. (2002). The substantive nature of psycholexical personality factors: 
a comparison across languages. Journal of Personality and Social Psychology, 83, 983-997. https:// doi.org/10.1037//0022-3514.83.4.983

Revelle, W. (2016). Using $R$ and the "psych" package to find $\omega$. Retrived from http://personality-project. org/r/psych/HowTo/omega.pdf

Revelle, W., \& Zinbarg, R. E. (2009). Coefficients alpha, beta, omega and the glb: Comments on Sijtsma. Psychometrika, 74, 145-154. https://doi.org/10.1007/ s11336-008-9102-z

Romero, E., Villar, P., \& López-Romero, L. (2015). Assessing six factors in Spain: Validation of the HEXACO-100 in relation on the Five Factor Model and other conceptually relevant criteria. Personality and Individual Differences, 76, 75-81. https:// doi.org/10.1016/j.paid.2014.11.056

Saucier, G. (1992). Openness versus intellect: Much ado about nothing? European Journal of Personality, 6, 381-386. https://doi.org/10.1002/per.2410060506

Saucier, G. (2009). Recurrent personality dimensions in inclusive lexical studies: Indications for a Big Six structure. Journal of Personality, 77, 1577-1614. https://doi.org/10.1111/j.1467-6494.2009.00593.x

Saucier, G., Hampson, S. E., \& Goldberg, L. R. (2000). Cross-language studies of lexical personality factors. In S. E. Hampson (Ed.), Advances in personality psychology (Vol. 1, pp. 1-36). Philadelphia, PA: Taylor \& Francis.

Saucier, G., \& Srivastava, S. (2015). What makes a good structural model of personality? Evaluating the Big Five and alternatives. In M. Mikulincer \& P. R. Shaver (Eds.), APA handbook of personality and social psychology. Vol. 4: Personality processes and individual differences (pp. 283-305). Washington, DC: APA.

Saucier, G., Thalmayer, A. G., Payne, D. L., Carlson, R., Sanogo, L. Ole-Kotikash, L., Church, A. T., Katigbak, M. S., Somer, O., Szarota, P., Szirmák, Z., \& Zhou, X. (2014). A basic bivariate structure of personality attributes evident across nine languages. Journal of Personality, 82, 1-14. https://doi. org/10.1111/jopy. 12028

Skimina, E., Cieciuch, J., \& Strus, W. (2018). Traits and values as predictors of the frequency of everyday behavior: Comparison between models and levels. Current Psychology. Advance online publication. https://doi.org/10.1007/s12144-018-9892-9

Soto, C. J., \& John, O. P. (2017). The next Big Five Inventory (BFI-2): Developing and assessing a hierarchical model with 15 facets to enhance bandwidth, fidelity, and predictive power. Journal of Personality and Social Psychology, 113, 117-143. https://doi.org/10.1037/pspp0000096

Strus, W., \& Cieciuch, J. (2019). Are the questionnaire and the psycho-lexical Big Twos the same? Towards an integration of personality structure within the Circumplex of Personality Metatraits. International Journal of Personality Psychology, 5, 18-35. https://doi.org/10.21827/ijpp.5.35594
Strus, W., Cieciuch, J., \& Rowiński, T. (2012, July). Polish version of Big Five Aspects Scales from International Personality Item Pool. Poster presented at the 16th European Conference on Personality, Trieste, Italy.

Szarota, P. (1996). Taxonomy of the Polish personality-descriptive adjectives of the highest frequency of use. Polish Psychological Bulletin, 27, 343-351.

Szarota, P., Ashton, M., \& Lee, K. (2007). Taxonomy and structure of the Polish personality lexicon. European Journal of Personality, 21, 823-852. https://doi.org/10.1002/per.635

Thalmayer, A. G., \& Saucier, G. (2014). The Questionnaire Big Six in 26 nations: Developing cross-culturally applicable Big Six, Big Five and Big Two inventories. European Journal of Personality, 28, 482-496. https://doi.org/10.1002/per. 1969

Thalmayer, A. G., Saucier, G., \& Eigenhuis, A. (2011). Comparative validity of brief to medium-length Big Five and Bix Six personality questionnaires. Psychological Assessment, 23, 995-1009. https:// doi.org/10.1037/a0024165

Thalmayer, A. G., Saucier, G., Ole-Kotikash, L., \& Payne, D. (2019). Personality structure in East and West Africa: Lexical studies of personality in Maa and Supyire-Senufo. Journal of Personality and Social Psychology. Advance online publication. https://doi.org/10.1037/pspp0000264

Thielmann, I., Akrami, N., Babarović, T., Belloch, A., Bergh, R., Chirumbolo, A., ... Lee, K. (2019). The HEXACO-100 across 16 languages: a large-scale test of measurement invariance. Journal of Personality Assessment. Advance online publication. https://doi.org/10.1080/00223891.2019.1614011

Trapnell, P. D. (1994). Openness versus intellect: a lexical left turn. European Journal of Personality, 8, 273-290. https://doi.org/10.1002/per.2410080405

Truskauskaitė-Kunevičienè, I., Kaniušonytė, G., Kratavičien, R., \& Kratavičiūtè-Ališauskienè, A. (2012). Psychometric properties of the Lithuanian versions of HEXACO-100 and HEXACO-60. Educational Psychology, 23, 6-14.

Wakabayashi, A. (2014). A sixth personality domain that is independent of the Big Five domains: The psychometric properties of the HEXACO Personality Inventory in a Japanese sample. Japanese Psychological Research, 56, 211-223. https://doi. org/10.1111/jpr.12045

Wilt, J., \& Revelle, W. (2019). The Big Five, everyday context and activities, and affective experience. Personality and Individual Differences, 136, 140-147. https://doi.org/10.1016/j.paid.2017.12.032 


\section{APPENDIX}

Table S1

Polish Items of the HEXACO-100 and HEXACO-60 Inventories

\begin{tabular}{|c|c|c|c|}
\hline No. & Scale & Subscale & Item \\
\hline $1^{*}$ & $\mathrm{O}$ & $\begin{array}{l}\text { Aesthetic } \\
\text { Appreciation }\end{array}$ & Wizyta w galerii sztuki byłaby dla mnie dość nudna. [R] \\
\hline 2 & $\mathrm{C}$ & Organization & Systematycznie sprzątam swoje miejsce pracy i/lub mieszkanie. \\
\hline $3^{*}$ & A & Forgiveness & Nie żywię urazy nawet wobec ludzi, którzy mnie bardzo skrzywdzili. \\
\hline $4^{*}$ & $\mathrm{x}$ & $\begin{array}{l}\text { Social } \\
\text { Self-Esteem }\end{array}$ & Zwykle czuję się z siebie zadowolony/a. \\
\hline $5^{*}$ & $\mathrm{E}$ & Fearfulness & Bał(a)bym się, gdybym musiał/a podróżować przy złej pogodzie. \\
\hline 6 & $\mathrm{H}$ & Sincerity & $\begin{array}{l}\text { Jeżeli czegoś chcę od osoby, której nie lubię, zachowuję się wobec niej } \\
\text { bardzo uprzejmie, aby to uzyskać. [R] }\end{array}$ \\
\hline $7^{*}$ & $\mathrm{O}$ & Inquisitiveness & $\begin{array}{l}\text { Chętnie dowiaduję się nowych rzeczy o historii i życiu politycznym } \\
\text { innych krajów. }\end{array}$ \\
\hline 8 & $\mathrm{C}$ & Diligence & W pracy często wyznaczam sobie ambitne cele. \\
\hline $9^{*}$ & A & Gentleness & Ludzie mówią mi czasami, że jestem zbyt krytyczny/a wobec innych. [R] \\
\hline $10^{*}$ & $\mathrm{X}$ & Social Boldness & Rzadko wyrażam swoje zdanie podczas spotkań grupowych. [R] \\
\hline $11^{*}$ & $\mathrm{E}$ & Anxiety & Czasem zamartwiam się drobiazgami i nic nie mogę na to poradzić. \\
\hline $12^{*}$ & $\mathrm{H}$ & Fairness & $\begin{array}{l}\text { Gdybym miał/a pewność, że nigdy nie zostanę złapany/a, był(a)bym } \\
\text { gotów/gotowa ukraść milion złotych. [R] }\end{array}$ \\
\hline 13 & $\mathrm{O}$ & Creativity & $\begin{array}{l}\text { Wolał(a)bym pracę wymagającą wykonywania rutynowych działań niż } \\
\text { taką, gdzie potrzeba kreatywności. [R] }\end{array}$ \\
\hline 14 & $\mathrm{C}$ & Perfectionism & $\begin{array}{l}\text { Często sprawdzam powtórnie wykonaną pracę, aby znaleźć ewentualne } \\
\text { błędy. }\end{array}$ \\
\hline $15^{*}$ & A & Flexibility & Ludzie czasami mówią mi, że jestem zbyt uparty/a. [R] \\
\hline 16 & $\mathrm{X}$ & Sociability & Unikam rozmów na błahe tematy. [R] \\
\hline $17^{*}$ & $\mathrm{E}$ & Dependence & $\begin{array}{l}\text { Kiedy cierpię z powodu jakiegoś bolesnego wydarzenia, potrzebuję } \\
\text { kogoś, kto sprawi, że poczuję się lepiej. }\end{array}$ \\
\hline $18^{*}$ & $\mathrm{H}$ & $\begin{array}{c}\text { Greed } \\
\text { Avoidance }\end{array}$ & Posiadanie dużych pieniędzy nie jest dla mnie szczególnie ważne. \\
\hline $19^{*}$ & $\mathrm{O}$ & $\begin{array}{l}\text { Unconven- } \\
\text { tionality }\end{array}$ & $\begin{array}{l}\text { Myślę, że poświęcanie uwagi niekonwencjonalnym ideom to strata } \\
\text { czasu. }[R]\end{array}$ \\
\hline $20^{*}$ & $\mathrm{C}$ & Prudence & $\begin{array}{l}\text { Decyzje podejmuję raczej pod wpływem chwili czy nastroju niż po } \\
\text { starannym namyśle. [R] }\end{array}$ \\
\hline $21^{*}$ & A & Patience & Ludzie uważają mnie za osobę wybuchową. [R] \\
\hline 22 & $\mathrm{X}$ & Liveliness & Prawie zawsze jestem pełen/pełna energii. \\
\hline $23^{*}$ & $\mathrm{E}$ & Sentimentality & Zbiera mi się na płacz, gdy widzę, jak inni płaczą. \\
\hline 24 & $\mathrm{H}$ & Modesty & Jestem zwyczajnym człowiekiem, wcale nie lepszym od innych. \\
\hline 25 & $\mathrm{O}$ & $\begin{array}{l}\text { Aesthetic } \\
\text { Appreciation }\end{array}$ & Nie poświęcił(a)bym czasu na czytanie tomiku poezji. [R] \\
\hline $26^{*}$ & $\mathrm{C}$ & Organization & $\begin{array}{l}\text { Planuję z odpowiednim wyprzedzeniem i tak wszystko organizuję, } \\
\text { aby uniknąć zamieszania w ostatniej chwili. }\end{array}$ \\
\hline
\end{tabular}

(Table S1 continues) 
Table S1

(Table S1 continued)

\begin{tabular}{|c|c|c|c|}
\hline No. & Scale & Subscale & Item \\
\hline $27^{*}$ & A & Forgiveness & $\begin{array}{l}\text { Mój stosunek do ludzi, którzy potraktowali mnie źle, to: „przebaczam } \\
\text { i zapominam”. }\end{array}$ \\
\hline 28 & $\mathrm{X}$ & $\begin{array}{l}\text { Social } \\
\text { Self-Esteem }\end{array}$ & Myślę, że większość ludzi lubi niektóre cechy mojego charakteru. \\
\hline 29 & $\mathrm{E}$ & Fearfulness & $\begin{array}{l}\text { Mógłbym/mogłabym wykonywać prace, które wiążą się } \\
\text { z niebezpieczeństwem. [R] }\end{array}$ \\
\hline $30 *$ & $\mathrm{H}$ & Sincerity & $\begin{array}{l}\text { Nie użył(a)bym pochlebstw, aby uzyskać podwyżkę czy awans w pracy, } \\
\text { nawet jeśli wydawałoby mi się to skuteczne. }\end{array}$ \\
\hline 31 & $\mathrm{O}$ & Inquisitiveness & Oglądanie map różnych miejsc sprawia mi przyjemność. \\
\hline $32^{*}$ & $\mathrm{C}$ & Diligence & Kiedy staram się osiągnąć jakiś cel, wytrwale do niego dążę. \\
\hline 33 & A & Gentleness & Jestem wyrozumiały/a dla błędów popełnianych przez innych. \\
\hline $34^{*}$ & $X$ & Social Boldness & W sytuacjach społecznych to ja zazwyczaj wychodzę z inicjatywą. \\
\hline $35^{*}$ & $\mathrm{E}$ & Anxiety & Martwię się różnymi rzeczami znacznie mniej niż większość ludzi. [R] \\
\hline 36 & $\mathrm{H}$ & Fairness & $\begin{array}{l}\text { Skusił(a)bym się na kupno kradzionej rzeczy, gdybym miał/a ograniczone } \\
\text { środki finansowe. [R] }\end{array}$ \\
\hline $37^{*}$ & $\mathrm{O}$ & Creativity & $\begin{array}{l}\text { Twórcza praca nad powieścią, piosenką czy obrazem sprawiałaby mi } \\
\text { przyjemność. }\end{array}$ \\
\hline $38^{*}$ & $\mathrm{C}$ & Perfectionism & Kiedy nad czymś pracuję, nie zwracam zbyt wiele uwagi na szczegóły. [R] \\
\hline $39^{*}$ & A & Flexibility & $\begin{array}{l}\text { Zwykle jestem dość ustępliwy/a i potrafię zmienić zdanie, gdy inni ludzie } \\
\text { się ze mną nie zgadzają. }\end{array}$ \\
\hline 40 & $X$ & Sociability & Lubię mieć wokół siebie dużo ludzi, z którymi mogę porozmawiać. \\
\hline $41^{*}$ & E & Dependence & $\begin{array}{l}\text { Potrafię radzić sobie z trudnymi sytuacjami, nie potrzebując } \\
\text { emocjonalnego wsparcia ze strony innych. [R] }\end{array}$ \\
\hline 42 & $\mathrm{H}$ & $\begin{array}{c}\text { Greed } \\
\text { Avoidance }\end{array}$ & Chciał(a)bym mieszkać w bardzo drogiej, ekskluzywnej okolicy. [R] \\
\hline $43^{*}$ & $\mathrm{O}$ & $\begin{array}{l}\text { Unconven- } \\
\text { tionality }\end{array}$ & Podobają mi się ludzie, którzy mają nietypowe poglądy. \\
\hline $44^{*}$ & $\mathrm{C}$ & Prudence & Popełniam dużo błędów, ponieważ działam bez zastanowienia. [R] \\
\hline 45 & A & Patience & Rzadko odczuwam złość, nawet gdy ludzie źle mnie traktują. \\
\hline $46^{*}$ & $X$ & Liveliness & Zazwyczaj jestem radosny/a i optymistyczny/a. \\
\hline 47 & $\mathrm{E}$ & Sentimentality & Kiedy ktoś, kogo dobrze znam, jest nieszczęśliwy, to niemal czuję jego ból. \\
\hline 48 & $\mathrm{H}$ & Modesty & $\begin{array}{l}\text { Nie chciał(a)bym, aby ludzie traktowali mnie jako kogoś, kto jest od nich } \\
\text { ważniejszy. }\end{array}$ \\
\hline $49^{*}$ & $\mathrm{O}$ & $\begin{array}{l}\text { Aesthetic } \\
\text { Appreciation }\end{array}$ & $\begin{array}{l}\text { Gdybym miał/a okazję, chętnie poszedłbym/poszłabym na koncert } \\
\text { muzyki klasycznej. }\end{array}$ \\
\hline 50 & $\mathrm{C}$ & Organization & $\begin{array}{l}\text { Ludzie często żartują sobie z bałaganu, jaki panuje w moim pokoju } \\
\text { czy na biurku. [R] }\end{array}$ \\
\hline 51 & A & Forgiveness & $\begin{array}{l}\text { Jeżeli ktoś raz mnie oszuka, zawsze będę w stosunku do niego } \\
\text { podejrzliwy/a. [R] }\end{array}$ \\
\hline $52^{*}$ & $X$ & $\begin{array}{l}\text { Social } \\
\text { Self-Esteem }\end{array}$ & Czuję, że nie jestem zbyt popularny/a w swoim środowisku. [R] \\
\hline
\end{tabular}

(Table S1 continues) 
Table S1

(Table $S 1$ continued)

\begin{tabular}{|c|c|c|c|}
\hline No. & Scale & Subscale & Item \\
\hline $53^{*}$ & $\mathrm{E}$ & Fearfulness & Gdy pojawia się fizyczne niebezpieczeństwo, jestem przerażony/a. \\
\hline $54^{*}$ & $\mathrm{H}$ & Sincerity & $\begin{array}{l}\text { Jeśli od kogoś czegoś chcę, śmieję się z jego żartów, nawet gdy są } \\
\text { zupełnie beznadziejne. [R] }\end{array}$ \\
\hline 55 & $\mathrm{O}$ & Inquisitiveness & Książka dotycząca historii nauki czy techniki bardzo by mnie znudziła. [R] \\
\hline 56 & $\mathrm{C}$ & Diligence & Często rezygnuję z wyznaczonego celu, zanim go osiągnę. [R] \\
\hline $57^{*}$ & A & Gentleness & Jestem raczej łagodny/a w ocenianiu innych ludzi. \\
\hline $58^{*}$ & $X$ & Social Boldness & $\begin{array}{l}\text { Będąc w grupie ludzi, często jestem tą osobą, która wypowiada się } \\
\text { w imieniu innych. }\end{array}$ \\
\hline 59 & $\mathrm{E}$ & Anxiety & Nie mam kłopotów ze snem wynikających ze stresu lub niepokoju. [R] \\
\hline $60^{*}$ & $\mathrm{H}$ & Fairness & $\begin{array}{l}\text { Nigdy nie przyjąłbym/przyjęłabym łapówki, nawet gdyby była bardzo } \\
\text { duża. }\end{array}$ \\
\hline $61^{*}$ & $\mathrm{O}$ & Creativity & Ludzie mówią, że mam bogatą wyobraźnię. \\
\hline $62^{*}$ & $\mathrm{C}$ & Perfectionism & $\begin{array}{l}\text { Zawsze staram się dokładnie wykonywać swoją pracę, nawet jeśli } \\
\text { pochłania to więcej czasu. }\end{array}$ \\
\hline $63^{*}$ & A & Flexibility & $\begin{array}{l}\text { Kiedy ludzie mówią mi, że nie mam racji, od razu zaczynam się z nimi } \\
\text { spierać. [R] }\end{array}$ \\
\hline $64^{*}$ & $x$ & Sociability & $\begin{array}{l}\text { Bardziej podoba mi się praca wymagająca stałego kontaktu z ludźmi niż } \\
\text { taka, w której pracuje się samemu. }\end{array}$ \\
\hline 65 & $\mathrm{E}$ & Dependence & $\begin{array}{l}\text { Za każdym razem, kiedy się czymś martwię, chcę podzielić się swoimi } \\
\text { troskami z drugą osobą. }\end{array}$ \\
\hline 66 & $\mathrm{H}$ & $\begin{array}{l}\text { Greed } \\
\text { Avoidance }\end{array}$ & $\begin{array}{l}\text { Chciał(a)bym, żeby ludzie widzieli, jak jeżdżę luksusowym } \\
\text { samochodem. [R] }\end{array}$ \\
\hline 67 & $\mathrm{O}$ & $\begin{array}{l}\text { Unconven- } \\
\text { tionality }\end{array}$ & Myślę, że jestem dość oryginalną osobą. \\
\hline 68 & $\mathrm{C}$ & Prudence & Nie pozwalam, żeby moim zachowaniem rządziły chwilowe impulsy. \\
\hline $69^{*}$ & A & Patience & Nie wpadam w złość tak szybko jak inni ludzie. \\
\hline 70 & $X$ & Liveliness & Ludzie często mówią, że mógłbym/mogłabym być weselszy/a. [R] \\
\hline $71^{*}$ & $\mathrm{E}$ & Sentimentality & Doświadczam silnych uczuć, kiedy ktoś bliski wyjeżdża na dłuższy czas. \\
\hline $72^{*}$ & $\mathrm{H}$ & Modesty & Myślę, że należy mi się większy szacunek niż zwykłej osobie. [R] \\
\hline 73 & $\mathrm{O}$ & $\begin{array}{l}\text { Aesthetic } \\
\text { Appreciation }\end{array}$ & Czasami lubię po prostu patrzeć na drzewa kołysane wiatrem. \\
\hline $74^{*}$ & $\mathrm{C}$ & Organization & $\begin{array}{l}\text { Moje niezorganizowanie jest powodem kłopotów, jakie miewam } \\
\text { w pracy. }[\mathrm{R}]\end{array}$ \\
\hline 75 & A & Forgiveness & Nie umiem całkowicie przebaczyć osobie, która zrobiła mi coś złego. [R] \\
\hline $76^{*}$ & $x$ & $\begin{array}{l}\text { Social } \\
\text { Self-Esteem }\end{array}$ & Czasem czuję, że jestem kimś bezwartościowym. [R] \\
\hline $77^{*}$ & $\mathrm{E}$ & Fearfulness & Nawet w nagłym wypadku nie wpadł(a)bym w panikę. [R] \\
\hline $78^{*}$ & $\mathrm{H}$ & Sincerity & Nie udawał(a)bym, że kogoś lubię, tylko po to, aby coś od tej osoby uzyskać. \\
\hline $79^{*}$ & $\mathrm{O}$ & Inquisitiveness & Nigdy nie lubiłem/am zaglądać do encyklopedii. [R] \\
\hline $80^{*}$ & $\mathrm{C}$ & Diligence & $\begin{array}{l}\text { W pracy ograniczam się jedynie do wykonywania niezbędnego } \\
\text { minimum. }[R]\end{array}$ \\
\hline
\end{tabular}


Table S1

(Table $S 1$ continued)

\begin{tabular}{|c|c|c|c|}
\hline No & Scale & Subscale & Item \\
\hline $81^{*}$ & A & Gentleness & Nawet jeśli ktoś popełnia dużo błędów, nie wyrażam się o nim negatywnie. \\
\hline 82 & $X$ & Social Boldness & Zwykle czuję się skrępowany/a, kiedy mówię coś do grupy ludzi. [R] \\
\hline 83 & E & Anxiety & Odczuwam silny niepokój, gdy oczekuję na jakąś ważną decyzję. \\
\hline $84^{*}$ & $\mathrm{H}$ & Fairness & $\begin{array}{l}\text { Uległ(a)bym pokusie używania fałszywych pieniędzy, gdybym miał(a) } \\
\text { pewność, że to nie wyjdzie na jaw. [R] }\end{array}$ \\
\hline $85^{*}$ & $\mathrm{O}$ & Creativity & Nie jestem typem osoby twórczej czy kreatywnej. [R] \\
\hline $86^{*}$ & $\mathrm{C}$ & Perfectionism & Ludzie często nazywają mnie perfekcjonist(k)ą. \\
\hline 87 & A & Flexibility & $\begin{array}{l}\text { Trudno mi pójść z kimś na kompromis, kiedy jestem przekonany/a, że to } \\
\text { ja mam rację. }[R]\end{array}$ \\
\hline $88^{*}$ & $\mathrm{X}$ & Sociability & $\begin{array}{l}\text { Pierwszą rzeczą, jaką zawsze robię w nowym miejscu, jest nawiązanie } \\
\text { bliższych kontaktów z ludźmi. }\end{array}$ \\
\hline 89 & $\mathrm{E}$ & Dependence & Rzadko mówię innym o swoich problemach. [R] \\
\hline $90^{*}$ & $\mathrm{H}$ & $\begin{array}{l}\text { Greed } \\
\text { Avoidance }\end{array}$ & $\begin{array}{l}\text { Posiadanie luksusowych i naprawdę kosztownych rzeczy sprawiałoby mi } \\
\text { mnóstwo przyjemności. [R] }\end{array}$ \\
\hline $91^{*}$ & $\mathrm{O}$ & $\begin{array}{l}\text { Unconven- } \\
\text { tionality }\end{array}$ & Uważam, że dyskusje o problemach filozoficznych są nudne. [R] \\
\hline $92^{*}$ & $\mathrm{C}$ & Prudence & $\begin{array}{l}\text { Wolę robić to, co mi akurat przychodzi do głowy, zamiast trzymać się } \\
\text { jakiegoś planu. }[\mathrm{R}]\end{array}$ \\
\hline 93 & A & Patience & Trudno mi utrzymać nerwy na wodzy, gdy ktoś mnie obraża. [R] \\
\hline $94^{*}$ & $\mathrm{X}$ & Liveliness & Większość ludzi jest bardziej entuzjastyczna i dynamiczna ode mnie. [R] \\
\hline $95^{*}$ & $\mathrm{E}$ & Sentimentality & $\begin{array}{l}\text { Nie ulegam uczuciom nawet w sytuacjach, w których większość ludzi } \\
\text { bardzo się wzrusza. [R] }\end{array}$ \\
\hline $96^{*}$ & $\mathrm{H}$ & Modesty & $\begin{array}{l}\text { Chciał(a)bym, żeby ludzie wiedzieli, że mam wysoką pozycję i jestem } \\
\text { kimś ważnym. [R] }\end{array}$ \\
\hline 97 & & Altruism & Współczuję ludziom, którym powiodło się w życiu gorzej niż mnie. \\
\hline 98 & & Altruism & Staram się hojnie wspomagać tych, którzy tego potrzebują. \\
\hline 99 & & Altruism & Nie miał(a)bym oporów, aby wyrządzić krzywdę komuś, kogo nie lubię. [R] \\
\hline 100 & & Altruism & Ludzie uważają mnie za osobę nieczułą. [R] \\
\hline
\end{tabular}

Note. *Item included in the HEXACO-60 inventory. [R] - reverse scored, $\mathrm{H}$ - Honesty-Humility, E - Emotionality, X - Extraversion, $\mathrm{A}$ - Agreeableness, $\mathrm{C}$ - Conscientiousness, $\mathrm{O}$ - Openness to Experience. 